\title{
The evolutionary status of the low-metallicity blue compact dwarf galaxy SBS 0940+544
}

\author{
N. G. Guseva ${ }^{1}$, Y. I. Izotov ${ }^{1}$, P. Papaderos ${ }^{2}$, F. H. Chaffee ${ }^{3}$, C. B. Foltz ${ }^{4}$, R. F. Green ${ }^{5}$, T. X. Thuan ${ }^{6}$, \\ K. J. Fricke ${ }^{2}$, and K. G. Noeske ${ }^{2}$ \\ 1 Main Astronomical Observatory, Ukrainian National Academy of Sciences, Golosiiv, Kyiv 03680, Ukraine \\ 2 Universitäts-Sternwarte, Geismarlandstraße 11, 37083 Göttingen, Germany \\ 3 W. M. Keck Observatory, 65-1120 Mamalahoa Hwy., Kamuela, HI 96743, USA \\ 4 MMT Observatory, University of Arizona, Tucson, AZ 85721, USA \\ 5 National Optical Astronomy Observatories, Tucson, AZ 85726, USA \\ 6 Astronomy Department, University of Virginia, Charlottesville, VA 22903, USA
}

Received 15 March 2001 / Accepted 22 August 2001

\begin{abstract}
We present the results of spectrophotometry and $V, R, I, \mathrm{H} \alpha \mathrm{CCD}$ photometry of the blue compact dwarf (BCD) galaxy SBS 0940+544. Broad-band images taken with the $2.1 \mathrm{~m} \mathrm{KPNO}{ }^{\star}$ and $1.23 \mathrm{~m}$ Calar Alto ${ }^{\star \star}$ telescopes reveal a compact high-surface-brightness $\mathrm{H}$ II region with ongoing star formation, located at the northwestern tip of the elongated low surface brightness (LSB) main body of the BCD. Very faint, patchy emission along the main body is seen in the $\mathrm{H} \alpha$ image. High signal-to-noise $4.5 \mathrm{~m}$ Multiple Mirror Telescope (MMT) ${ }^{\star \star \star}$ and $10 \mathrm{~m}$ Keck II telescope ${ }^{\dagger}$ long-slit spectroscopy of SBS $0940+544$ is used to derive element abundances of the ionized gas in the brightest $\mathrm{H}$ II region and to study the stellar population in the host galaxy. The oxygen abundance in the brightest region with strong emission lines is $12+\log (\mathrm{O} / \mathrm{H})=7.46-7.50$, or $1 / 29-1 / 26$ solar, in agreement with earlier determinations and among the lowest for BCDs. $\mathrm{H} \beta$ and $\mathrm{H} \alpha$ emission lines and $\mathrm{H} \delta$ and $\mathrm{H} \gamma$ absorption lines are detected in a large part of the main body. Three methods are used to put constraints on the age of the stellar population at different positions along the major axis. They are based on (a) the equivalent widths of the emission lines, (b) the equivalent widths of the absorption lines and (c) the spectral energy distributions (SED). Several scenarios of star formation have been considered. We find that models with single instantaneous bursts cannot reproduce the observed SEDs, implying that star formation in the main body of SBS 0940+544 was continuous. The observed properties in the main body can be reproduced by a continuous star formation process which started not earlier than $100 \mathrm{Myr}$ ago, if a small extinction is assumed. However, the observations can be reproduced equally well by a stellar population forming continuously since $10 \mathrm{Gyr}$, if the star formation rate has increased during last $100 \mathrm{Myr}$ in the main body of SBS $0940+544$ by at least a factor of five. We also investigate the age of the reddest southern region of the main body, where no absorption and emission lines are detected. On the assumption of zero extinction in this faint region, the observed spectrum can be fitted by a theoretical SED of a stellar population continuously formed with a constant star formation rate between $100 \mathrm{Myr}$ and 10 Gyr ago. If, however, a small extinction of $C(\mathrm{H} \beta) \sim 0.1$ is present in this region then the observed spectrum can be fitted by a theoretical SED of a stellar population continuously formed between $100 \mathrm{Myr}$ and 1 Gyr ago with a constant star formation rate. However, the poor signal-to-noise ratio of the spectrum and large photometric errors preclude reliable determination of the age of the southern region. In summary, we find no compelling evidence which favors either a young or an old age of SBS 0940+544.
\end{abstract}

Key words. galaxies: fundamental parameters - galaxies: starburst - galaxies: abundances - galaxies: photometry - galaxies: individual (SBS 0940+544)

\footnotetext{
Send offprint requests to: N. G. Guseva,

e-mail: guseva@mao.kiev.ua

* Kitt Peak National Observatory (KPNO) is operated by the Association of Universities for Research in Astronomy, Inc., under cooperative agreement with the National Science Foundation.

** German-Spanish Astronomical Center, Calar Alto, operated by the Max-Planck-Institute for Astronomy, Heidelberg, jointly with the Spanish National Commission for Astronomy.
}

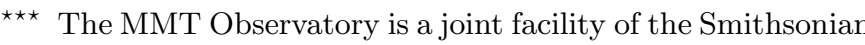
Institution and the University of Arizona.

$\dagger$ W. M. Keck Observatory is operated as a scientific partnership among the California Institute of Technology, the University of California and the National Aeronautics and Space Administration. The Observatory was made possible by the generous financial support of the W. M. Keck Foundation.
} 


\section{Introduction}

Blue compact dwarf (BCD) galaxies are characterized by ongoing intense star formation (SF) activity as shown by strong nebular emission lines superimposed on a blue continuum (e.g. Sargent \& Searle 1970; Lequeux et al. 1979; Kunth \& Sargent 1983; Izotov et al. 1994). They are compact and metal-deficient objects with oxygen abundances ranging between $1 / 50$ and $1 / 3$ solar (e.g. Izotov \& Thuan 1999) and possess large amounts of neutral gas (Thuan \& Martin 1981; van Zee et al. 1998; Thuan et al. 1999b).

Most of the BCDs studied so far have revealed extended low surface brightness red stellar sheets underlying the blue star-forming regions (Thuan 1983; Loose \& Thuan 1986). Some of these stellar sheets have been resolved with the Hubble Space Telescope (HST) into red giant stars (Schulte-Ladbeck; Crone \& Hopp 1998; Crone et al. 2000). This indicates the presence of an evolved lowmass stellar population in the underlying host galaxies.

The tiny fraction of galaxies with extremely low oxygen abundance in the ionized gas (less than $\left.1 / 20(\mathrm{O} / \mathrm{H})_{\odot}\right)$ has been suggested by Izotov \& Thuan (1999) to be young galaxies, based on chemical element abundance arguments. Nearly ten such galaxies with good abundance determinations are known to date. The outer parts of these galaxies are blue. The spectral energy distributions and colours of their outskirts are consistent with those of stellar populations with ages less than a few $100 \mathrm{Myr}$ (see the examples of SBS 0335-052 (Izotov et al. 1997b; Thuan et al. 1997; Papaderos et al. 1998) and SBS $1415+437$ (Thuan et al. 1999a)).

Detailed spectroscopic and photometric studies of extremely metal-poor BCDs based on observations with large telescopes are useful to shed light on the origin of these galaxies, deduce their star formation history and constrain their ages.

A good candidate for a young galaxy is SBS 0940+544. This galaxy was discovered in the course of the Second Byurakan Survey (SBS, Markarian et al. 1983) and described as a compact extragalactic $\mathrm{H}$ II region without an underlying host galaxy, with strong emission lines, a weak continuum and an ultraviolet excess.

A first abundance determination in the brightest $\mathrm{H}$ II region of SBS $0940+544$ was made by Izotov et al. (1991) who derived an oxygen abundance $12+\log (\mathrm{O} / \mathrm{H})=$ $7.52 \pm 0.12$ and a helium mass fraction $Y=0.246 \pm 0.030$. Izotov et al. (1997c), using higher $S / N$ ratio observations, subsequently derived a lower value of $12+\log (\mathrm{O} / \mathrm{H})=$ $7.43 \pm 0.01$. Because of its very low metal content, SBS $0940+544$ was used for the determination of the primordial helium abundance (Izotov et al. 1994; Izotov \& Thuan 1998b) and to study heavy element abundances (Thuan et al. 1995; Izotov \& Thuan 1999). The galaxy also has been studied spectroscopically by Augarde et al. (1994) and Comte et al. (1994), without an element abundance determination.
$B$ and $R$ surface photometry and colour profiles of SBS 0940+544 have been derived by Doublier et al. (1997) (see also Doublier et al. 1999).

Correcting the $21 \mathrm{~cm}$ heliocentric velocity $V=$ $1638 \mathrm{~km} \mathrm{~s}^{-1}$ for Virgocentric infall with a velocity of $220 \mathrm{~km} \mathrm{~s}^{-1}$ and adopting a Hubble constant of $75 \mathrm{~km} \mathrm{~s}^{-1} \mathrm{Mpc}^{-1}$, Thuan et al. (1999b) derived a distance of $D=26.7 \mathrm{Mpc}$ for SBS $0940+544$. At this distance, assumed here throughout, $1^{\prime \prime}$ corresponds to a projected linear scale of $129 \mathrm{pc}$. The total neutral hydrogen $\mathrm{H}$ I mass in SBS $0940+544$ is $\log \left(M(\mathrm{H} \mathrm{I}) / M_{\odot}\right)=8.71($ or $M(\mathrm{H} \mathrm{I})=$ $\left.5.1 \times 10^{8} M_{\odot}\right)($ Thuan et al. 1999b).

In this paper we present new $V, R, I$ and $\mathrm{H} \alpha$ photometry and very high signal-to-noise spectroscopic observations of SBS $0940+544$. We use those data to study the stellar populations in the BCD, put constraints on its age and derive elemental abundances in the ionized gas, including the helium abundance. In Sect. 2 we describe the observations and data reduction. The photometric properties of SBS $0940+544$ are discussed in Sect. 3. We derive elemental abundances in the brightest $\mathrm{H}$ II region in Sect. 4. In Sect. 5 the stellar populations are discussed and constraints on the age of SBS $0940+544$ are considered. Section 6 summarizes the results.

\section{Observations and data reduction}

\subsection{Photometry}

Direct images of SBS 0940+544 were acquired in two observing runs. Broad-band Johnson $V$ and Cousins $I$ images and narrow-band images in the $\mathrm{H} \alpha$ line at $\lambda 6606 \AA$ with a full width at half maximum $(F W H M)$ through a passband of $78 \AA$ and in the adjacent continuum at $\lambda 6520 \AA$ through a passband with $F W H M=84 \AA$ were obtained with the Kitt Peak $2.1 \mathrm{~m}$ telescope on April 18 and April 22, 1999, respectively. The telescope was equipped with a $1 \mathrm{k}$ Tektronix CCD detector operating at a gain of $3 \mathrm{e}^{-} \mathrm{ADU}^{-1}$, giving an instrumental scale of 0.305 pixel $^{-1}$ and field of view of $5^{\prime}$. The total exposure of 40 and $60 \mathrm{~min}$ in $V$ and $I$, respectively, was split into four subexposures, each being slightly offset from the others. Likewise, narrow-band exposures in the $\mathrm{H} \alpha$ line and the adjacent continuum bluewards of $\mathrm{H} \alpha$ were taken for $25 \mathrm{~min}$ each. The seeing during the broad- and narrowband observations was $F W H M 1$ 1'2 and 2'.2, respectively.

Another series of exposures with a total integration time of 2.5 hours in Cousins $R$ was taken with the $1.23 \mathrm{~m}$ telescope at Calar Alto in the period January 24 to February 22, 2000. The telescope was equipped with a $2 \mathrm{k}$ SITe detector at its Cassegrain focus giving a usable field of view of $\sim 11^{\prime}$ and an instrumental scale of $00^{\prime \prime} 5$ pixel $^{-1}$. The $F W H M$ of point sources in the coadded $R$ exposures is $\approx 1^{\prime \prime} .5$.

Bias and flat-field frames were obtained during each night of both observing runs. Broad-band images were calibrated by observing different standard fields from Landolt (1992). Our calibration uncertainties are estimated to 

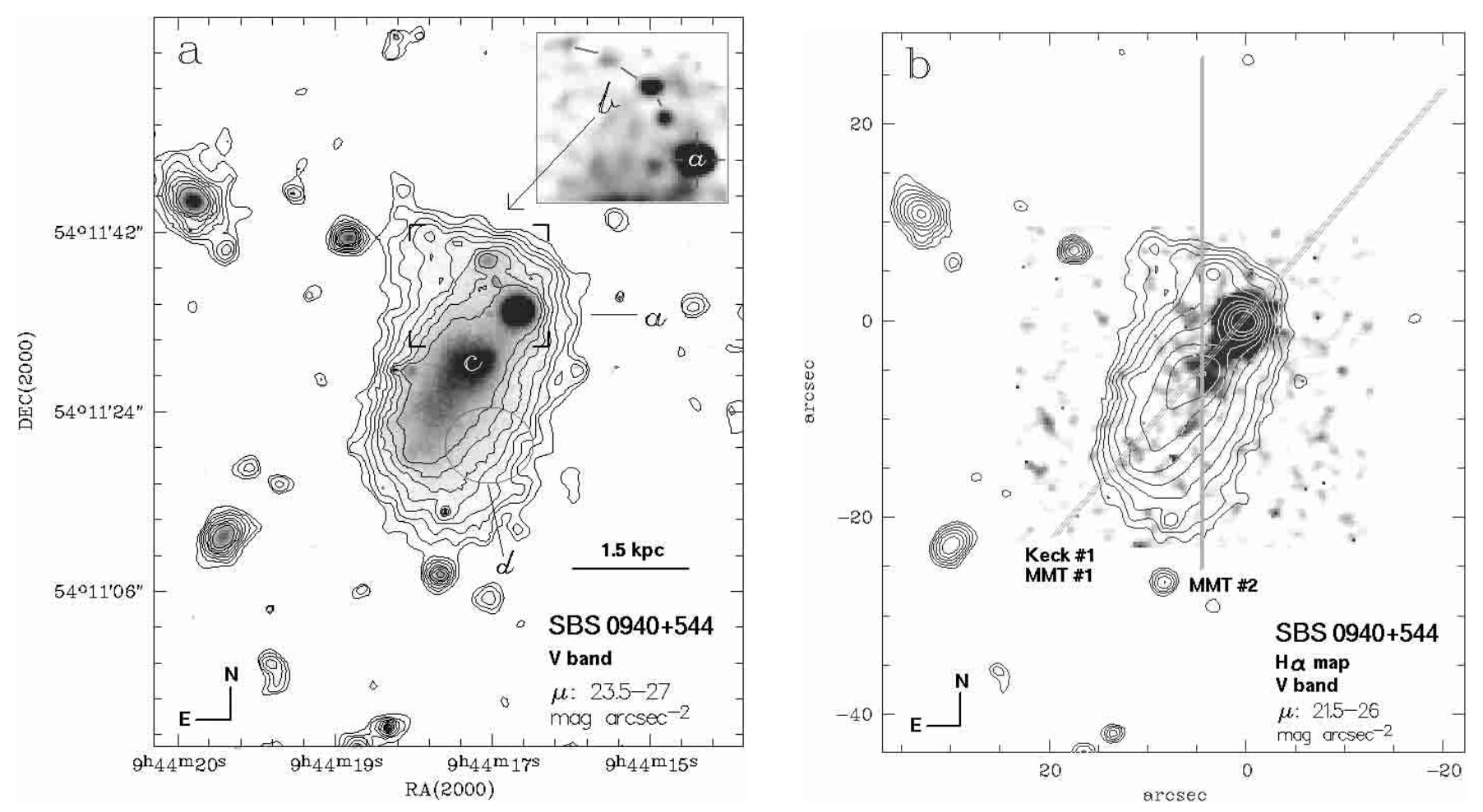

Fig. 1. a) $V$ image of the cometary iI BCD SBS $0940+544$. The overlayed contours correspond to surface brightness levels between $23.5 \mathrm{mag} \operatorname{arcsec}^{-2}$ and $27 \mathrm{mag} \operatorname{arcsec}^{-2}$ in steps of $0.5 \mathrm{mag}$. The inset at the upper right shows a deconvolved $13^{\prime \prime} \times$ $11^{\prime \prime}$ subframe at the northern tip of the galaxy. Adjacent to the brightest H II region $a$ is a chain of other compact sources (labeled $b$ traceable to $\sim 10^{\prime \prime}$ northeast of $a$. From the continuum-subtracted H $\alpha$ map of SBS 0940+544 (right panel) and from the spectroscopic study (Sect. 4), star-formation activity is found to be also present close to the optical maximum of the LSB body of the BCD (labeled $c$ ). The average $V-R$ and $V-I$ colours of the main body increase from $\sim 0.16$ mag and $\sim 0.43 \mathrm{mag}$ in region $c$ to $\sim 0.34 \mathrm{mag}$ and $\sim 0.65 \mathrm{mag}$ in region $d$. b) Continuum-subtracted H $\alpha$ map of SBS $0940+544$ overlayed with $V$ band contours between $21.5 \mathrm{mag} \operatorname{arcsec}^{-2}$ and $26 \mathrm{mag}_{\operatorname{arcsec}^{-2}}$ in steps of 0.5 mag. Roughly $80 \%$ of the H $\alpha$ emission originates from the compact $\mathrm{H}$ II region $a$. The long-slit positions during the two observations with the Multiple Mirror Telescope (MMT \#1 and MMT \#2) and with the Keck II telescope (Keck \#1) are indicated.

be well below 0.05 mag in all bands. Standard reduction steps, including bias subtraction, flat-field correction and absolute flux calibration were carried out using the IRAF ${ }^{1}$ and ESO MIDAS ${ }^{2}$.

\subsection{Spectroscopy}

High $S / N$ long-slit spectroscopy of SBS 0940+544 was obtained with the $4.5 \mathrm{~m}$ Multiple Mirror Telescope (MMT) and with the $10 \mathrm{~m}$ Keck II telescope over a wavelength range $3600-7500 \AA$.

The first set of observations was carried out with the MMT at two slit positions (see Fig. 1b). Observations were made with the blue channel of the MMT spectrograph operated with a $3072 \times 1024$ CCD detector. A $1^{\prime \prime} .5 \times 180^{\prime \prime}$ slit was used. The spatial scale along the slit was $00^{\prime \prime} 3$ pixel $^{-1}$, with a spectral resolution of $\sim 7 \AA(F W H M)$. The final

\footnotetext{
${ }^{1}$ IRAF is the Image Reduction and Analysis Facility distributed by the National Optical Astronomy Observatory, which is operated by the Association of Universities for Research in Astronomy (AURA) under cooperative agreement with the National Science Foundation (NSF).

2 Munich Image Data Analysis System, provided by the European Southern Observatory (ESO).
}

spatial scale is $0^{\prime \prime} 6$ pixel $^{-1}$ after binning every two consecutive rows into one.

The first slit orientation with position angle $\mathrm{PA}=$ $-41^{\circ}$ (MMT \#1, cf. Fig. 1b), was centered on the brightest star-forming $\mathrm{H}$ II region (denoted as $a$ in Fig. 1a) and oriented along the elongated main body of the galaxy close to the direction of the major axis. This 120-min spectrum was obtained on December 13, 1996 at an airmass of 1.2. The total exposure time was broken into four $30 \mathrm{~min}$ exposures.

The second slit orientation (MMT \#2, cf. Fig. 1b) is in the $\mathrm{N}-\mathrm{S}$ direction with $\mathrm{PA}=0^{\circ}$, centered on the second brightest region, denoted as $c$ in Fig. 1a. These observations were carried out on December 12, 1996 with an exposure time of $120 \mathrm{~min}$ at an airmass of 1.2. The total exposure time was also broken into four 30 min exposures. The seeing during both sets of MMT observations was 0.' 8 .

The Keck II observations were carried out on January 9, 2000 with the low-resolution imaging spectro-

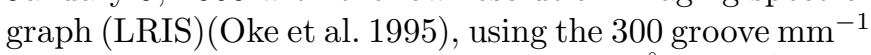
grating which provides a dispersion of $2.52 \AA$ pixel $^{-1}$ and a spectral resolution of about $8 \AA$ in the first order. The slit was $1^{\prime \prime} \times 180^{\prime \prime}$, centered on the brightest $\mathrm{H}$ II region and oriented with a position angle $\mathrm{PA}=-41^{\circ}$, the same as during 
the first set of MMT observations. No binning along the spatial axis has been done, yielding a spatial sampling of $0^{\prime \prime} 2$ pixel $^{-1}$. The total exposure time was $60 \mathrm{~min}$, broken into three $20 \mathrm{~min}$ exposures. All exposures were taken at an airmass of 1.2. The seeing was $00^{\prime \prime} 9$.

Signal-to-noise ratios $S / N \sim 50$ and $\sim 100$ were reached in the continuum near $\mathrm{H} \beta$ for the brightest part of the galaxy during the MMT and Keck II observations. No correction for atmospheric dispersion was made because of the small airmass during all observations. This effect, although small, may introduce some uncertainties in the flux determination of the weaker lines. At an airmass of 1.2 and position angle $-41^{\circ}$ the shift of the emitting region at the $[\mathrm{O}$ II $] \lambda 3727$ line relative to the same region at the $[\mathrm{O}$ III $] \lambda 5007$ line in the direction perpendicular to the slit is $0 . \prime 4$ (Filippenko 1982). The shift at [O III] $\lambda 4363$ is much smaller, only $0{ }^{\prime \prime} 1$. In Sect. 4 we estimate the uncertainties introduced by this effect on the abundance determination. Spectra of a He-Ne-Ar comparison lamp were obtained after each exposure during the MMT observations, and spectra of a $\mathrm{Hg}$-Ne-Ar comparison lamp were obtained after each exposure during the Keck II observations. They were used for wavelength calibration. Two spectrophotometric standard stars, Feige 34 and HZ 44, were observed for flux calibration. The data reduction was made with the IRAF software package. The two-dimensional spectra were bias-subtracted and flat-field corrected. Cosmicray removal, wavelength calibration, night sky background subtraction, correction for atmospheric extinction and absolute flux calibration were then performed.

One-dimensional spectra for the bright $\mathrm{H}$ II region $a$ of SBS $0940+544$ were extracted from the Keck II and MMT data within large apertures of $1^{\prime \prime} \times 4^{\prime \prime}$ and $1^{\prime \prime} \cdot 5 \times$ $3^{\prime \prime}$ respectively to minimize the effect of atmospheric dispersion (Fig. 5). In addition we extracted spectra showing hydrogen Balmer absorption lines for four regions along the main body of the galaxy (i.e. along the slit oriented at $\mathrm{PA}=-41^{\circ}$ ). The selected regions, denoted 1 to 4 (Keck II observations) and 1a to 4a (MMT) in Tables 4 and 5 were at adjacent positions along the major axis of the BCD, with the origin taken to be at the center of region $a$. Regions 1 and 1a coincide with region $c$ in Fig. 1a. The spatial extents of the 4 regions along the slit were $33^{\prime \prime} 0$, $22^{\prime \prime} 4,4{ }^{\prime \prime} 8$ and $33^{\prime \prime} 6$ for the MMT observations and $33^{\prime \prime} 0,22^{\prime \prime} 6$, 3 "' 6 and 4 ". 8 for the Keck II observations. Additionally, spectra with apertures $10{ }^{\prime \prime} 8$ and 7 '.2 centered on region $c$ were extracted from the two-dimensional MMT spectrum obtained at the position angle $\mathrm{PA}=0^{\circ}$ (regions 5 and 6 in the Tables 4 and 5).

\section{Photometric analysis}

\subsection{Morphology and colour distribution}

Star formation activity in SBS $0940+544$ takes place primarily in the unresolved high-surface-brightness $\mathrm{H}$ II region labeled $a$ in Fig. 1a. This region with an absolute $V$ magnitude of -13.6 mag accounts for $80 \%$ of the total
$\mathrm{H} \alpha$ emission and for nearly $1 / 4$ of the total $V$ light of the BCD within the $25 \mathrm{~V}$ mag $\operatorname{arcsec}^{-2}$ isophote. A chain of faint point-like sources superimposed on an asymmetric curved arm originating from region $a$ is traceable to $\sim 10^{\prime \prime}$ further to the northeast. This feature, labeled $b$ in Fig. 1a, is not detected in $\mathrm{H} \alpha$. Another feature is marginally visible to the SW side of the elongated low-surface-brightness (LSB) main body of the galaxy, at a surface brightness level $\gtrsim 25.5 \mathrm{~V} \mathrm{mag} \operatorname{arcsec}^{-2}$ (region southwards of feature $d$ in Fig. 1a). Judging from the continuum-subtracted $\mathrm{H} \alpha$ map (Fig. 1b), star formation in SBS $0940+544$ is not exclusively confined to region $a$ but also occurs in the LSB host. A secondary $\mathrm{H} \alpha$-emitting region is visible close to the geometrical center of the outer isophotes (region $c$ in Fig. 1a).

Evidence of recent star-formation activity along the major axis of the cometary LSB body of the BCD is also seen in the $V-I$ and $V-R$ colour maps (Fig. 2). The $V-I$ and $V-R$ colours in region $c$ are respectively $\sim 0.43 \mathrm{mag}$ and $\sim 0.16 \mathrm{mag}$. The $V-R$ colour index remains nearly constant at $\sim 0.2 \mathrm{mag}$ over a large part of the LSB body out to its southeastern extreme end (position $+13^{\prime \prime},-13^{\prime \prime}$ in Fig. $2 \mathrm{~b}$ ) and increases to $\sim 0.34 \mathrm{mag}$ at the faint $\left(\mu_{V} \approx 24.0-24.5 \mathrm{mag} \operatorname{arcsec}^{-2}\right)$ southern region $d$ (position $\sim+5^{\prime \prime},-14^{\prime \prime}$ ). The $V-I$ colour shows a similar general, although less well-defined trend, varying from $\sim 0.4-0.5 \mathrm{mag}$ in the main body to $\sim 0.65 \mathrm{mag}$ in region $d$ and getting bluer again (0.36 mag) close to the southeastern tip of the LSB host. The smooth colour change with decreasing intensity in the LSB body is accompanied by a clear isophotal twist between 23.5 and $27 \mathrm{~V}$ mag $\operatorname{arcsec}^{-2}$, with the position angle PA changing from $-40^{\circ}$ to $-10^{\circ}$ and an average gradient of $8.5^{\circ} \mathrm{mag}^{-1}$. Likewise, the ellipticity $10 \times\left(1-\frac{b}{a}\right)$ of the LSB host, $a$ and $b$ denoting respectively the semimajor and semiminor axis of the ellipse fitted to a given isophote, decreases from 5.5 at $24 \mathrm{~V}$ mag $\operatorname{arcsec}^{-2}$ to 3.9 at $27 \mathrm{~V}$ mag $\operatorname{arcsec}^{-2}$.

Region $a$ shows extraordinarily blue colours: $V-I=$ -0.5 mag and $V-R=-0.2$ mag. Doublier et al. (1997) have obtained $B$ and $R$ photometry of SBS $0940+544$. They mistook region $a$ for a Galactic foreground star. Spectra of the object show without doubt that it is an extremely compact $\mathrm{H}$ II region with very strong emission lines (Fig. 5) at the redshift of SBS 0940+544. Izotov et al. (1991) first derived element abundances in this H II region, followed by Izotov et al. (1994) who named it SBS $0940+544 \mathrm{~N}$.

\subsection{Surface photometry and colours}

As remarked in Sect. 3.1 (see also Sects. 4 and 5), signs of low-level ongoing or recent star formation are present all over the inner part of the main body of SBS $0940+544$. In order to disentangle the stellar from the gaseous emission and better constrain the surface brightness and colour distribution of its faint underlying LSB host, we performed surface photometry in all available broad-band filters in 

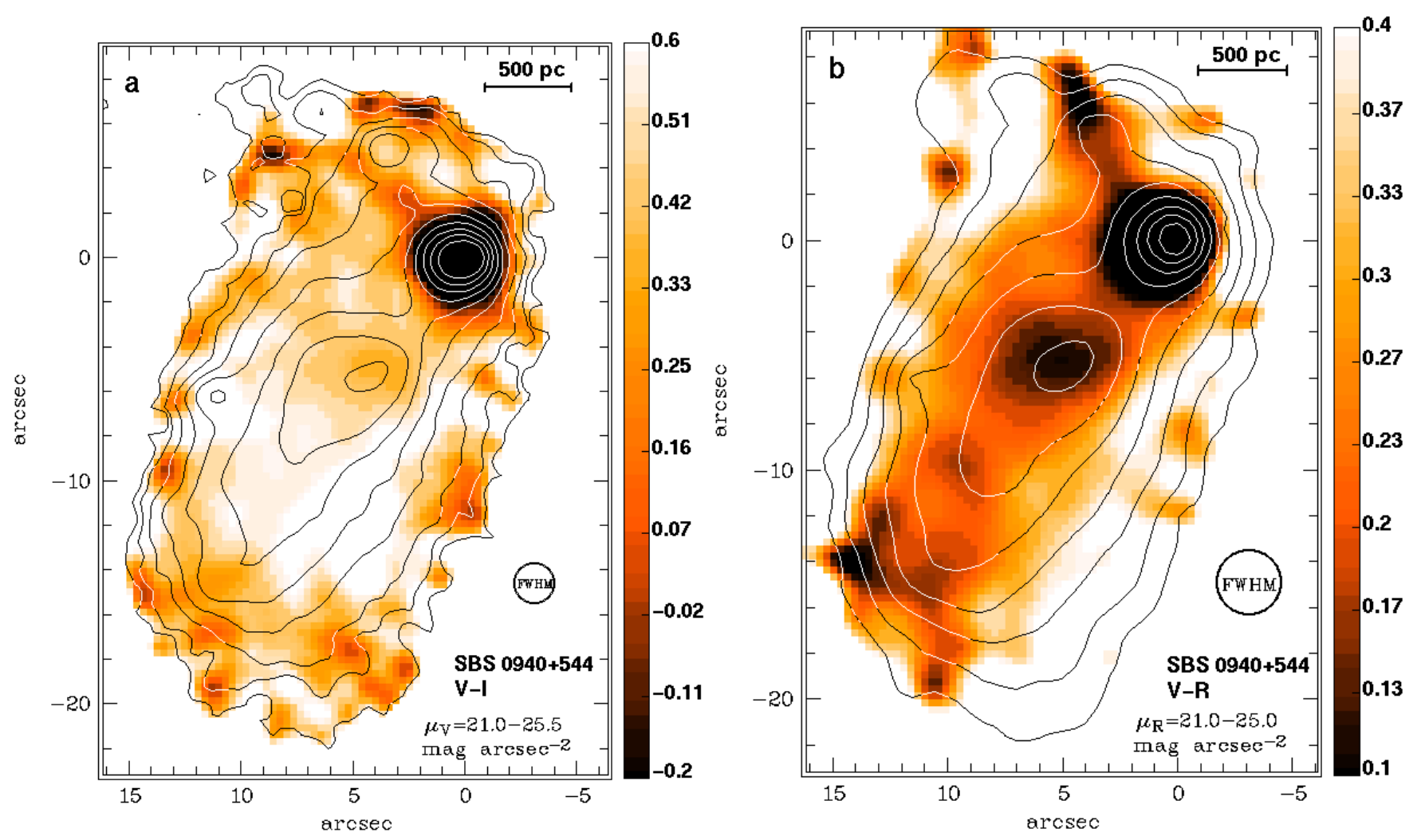

Fig. 2. a) $V-I$ map of SBS $0940+544$ displayed in the colour range -0.2 to +0.6 mag. The overlayed contours are from 21.0 to $25.5 \mathrm{~V}$ mag $\operatorname{arcsec}^{-2}$ in steps of $0.5 \mathrm{mag}$. b) $V-R$ map of the same region of the BCD displayed in the colour interval 0.1 to $0.4 \mathrm{mag}$. The overlayed contours are from 21.0 to $25.0 R \mathrm{mag} \operatorname{arcsec}^{-2}$ in steps of $0.5 \mathrm{mag}$. The bluest region in either map coincides with the northwestern $\mathrm{H}$ II region $a$, with an average $V-I$ and $V-R$ of $\lesssim-0.5$ mag and -0.2 mag, respectively. The angular resolution of each map is indicated by a circle, the diameter of which is equal to the $F W H M$ of point sources in the corresponding smoothed input images used for its derivation (1". 8 and $2^{\prime \prime} .75$ for the $V-I$ and $V-R$ map, respectively).

two steps. First we fitted the intensity distribution and subtracted both the dominant region $a$ and all adjacent point sources (designated by $b$, cf. Fig. 1a). We then derived surface brightness profiles (SBPs) for the main body using methods described in Papaderos et al. (1996b). SBPs computed in this way (Fig. 3a) trace the luminosity of the cometary LSB host of the BCD and the superimposed star-forming zone extending from region $c$ to the SE corner. The radial intensity distribution of the LSB host was approximated by adjusting different fitting laws to the outer part of the SBPs. We then computed SBPs for the whole BCD, and by subtracting from them the best model for the intensity distribution of the LSB host, we obtained the surface brightness distribution of the discrete and diffuse starburst sources located in the northwestern part and main body (cf. Fig. 3b).

The SBPs of the main body (Fig. 3a) provide an upper limit to the intensity distribution of the underlying unresolved older stellar population of the LSB host, since part of the light for $R^{*} \lesssim 8^{\prime \prime}$ comes from region $c$ and adjacent young sources. An exponential fitting law provides a reasonable approximation to the SBPs for $\mu_{V} \gtrsim 24.5 \mathrm{mag} \operatorname{arcsec}^{-2}$, for radii $<10^{\prime \prime}$, however, it predicts a higher intensity than the one observed. This is also the case for the SBPs derived for the total emission of the BCD (Fig. 3b). This type of convex profile with an exponential distribution in the outer parts, and leveling off in the inner part (within 1-3 scale lengths) is not rare among low-luminosity dwarf ellipticals (e.g. Binggeli \& Cameron 1991), dwarf irregulars (Patterson \& Thuan 1996; Makarova 1999; van Zee 2000) and blue low surface brightness galaxies (Rönnback \& Bergvall 1994; Bergvall et al. 1999), and has been reported in a few BCDs (e.g. Vennik et al. 1996; Papaderos et al. 1996b; Telles et al. 1997; Papaderos et al. 1999; Fricke et al. 2001). The observed profile shape suggests that a Sérsic (1990) profile of the form

$I\left(R^{*}\right)=I_{0} \exp \left(-\frac{R^{*}}{\alpha}\right)^{\eta}$

with $\eta>1$ provides a better approximation to the light of the LSB host than a pure exponential $(\eta=1)$.

An alternative fitting formula which well reproduces an exponential distribution flattening inwards has been discussed by Papaderos et al. (1996b):

$I\left(R^{*}\right)=I_{0} \exp \left(-\frac{R^{*}}{\alpha}\right)\left[1-q \exp \left(-P_{3}\left(R^{*}\right)\right)\right]$

where $P_{3}\left(R^{*}\right)$ is

$P_{3}\left(R^{*}\right)=\left(\frac{R^{*}}{b \alpha}\right)^{3}+\left(\frac{R^{*}}{\alpha} \frac{1-q}{q}\right)$. 

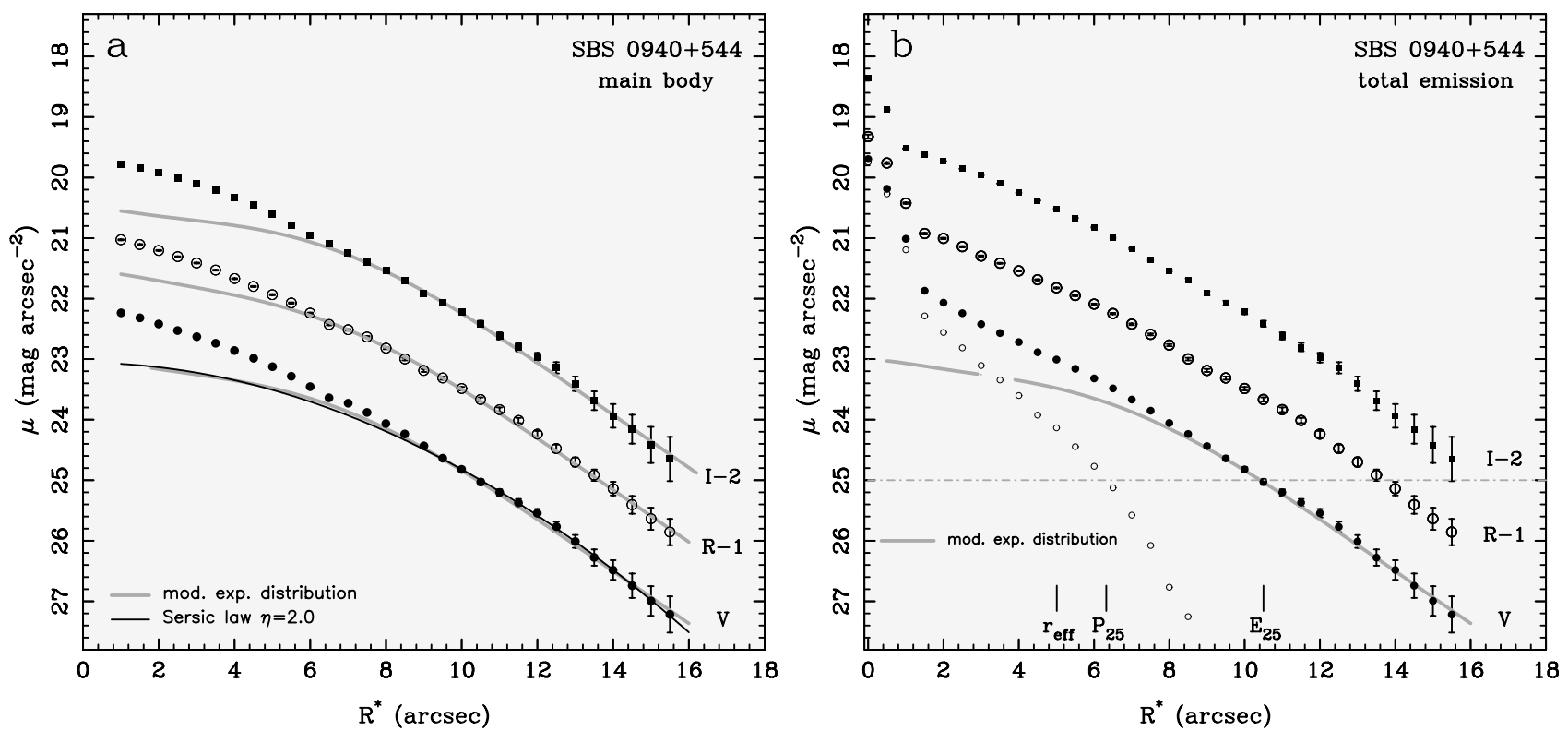

Fig. 3. a) Surface brightness profiles (SBPs) of the main body of SBS $0940+544$ after subtraction of the brightest $H$ II region $a$ and the adjacent point sources $b$ (cf. Fig. 1a). For the sake of clarity, the $R$ and $I$ SBPs have been shifted vertically by -1 and -2 mag respectively. The modeled surface brightness distributions of the underlying LSB host in the $V$ band corresponding to a Sérsic fitting law with an exponent $\eta=2$ (cf. Eq. (1)) and a modified exponential distribution with $(b, q)=(3.0,0.9)$ (Eq. (2)) are shown respectively by the thin/black and thick/grey curves. b) SBPs of the total emission of SBS $0940+544$. The meaning of the symbols is the same as in the left panel. Small open circles show the surface brightness distribution of the light in excess of the model for the LSB host (thick/grey curve) in the $V$ band. The effective radius $r_{\text {eff }}$ of the $V$ band SBP and the isophotal radii $P_{25}$ and $E_{25}$ of the starburst and LSB component at $25 \mathrm{~V}$ mag $\operatorname{arcsec}^{-2}$ are indicated.

Table 1. Structural properties of the starburst - and LSB component of SBS $0940+544$.

\begin{tabular}{lccccccccccc}
\hline Band & $\begin{array}{c}\mu_{\mathrm{E}, 0} \\
\mathrm{mag} \mathrm{arcsec}\end{array}$ & $\begin{array}{c}\alpha \\
\operatorname{arcsec}\end{array}$ & $\begin{array}{c}m_{\mathrm{LSB}}^{\mathrm{fit}} \\
\mathrm{mag}\end{array}$ & $\begin{array}{c}P_{25} \\
\mathrm{kpc}\end{array}$ & $\begin{array}{c}m_{P_{25}} \\
\mathrm{mag}\end{array}$ & $\begin{array}{c}E_{25} \\
\mathrm{kpc}\end{array}$ & $\begin{array}{c}m_{E_{25}} \\
\mathrm{mag}\end{array}$ & $\begin{array}{c}m_{\mathrm{LSB}} \\
\mathrm{mag}\end{array}$ & $\begin{array}{c}m_{\mathrm{SBP}} \\
\mathrm{mag}\end{array}$ & $\begin{array}{c}m_{\mathrm{tot}} \\
\mathrm{mag}\end{array}$ & $\begin{array}{c}r_{\mathrm{eff}}, r_{80} \\
\mathrm{kpc}\end{array}$ \\
$(1)$ & $(2)$ & $(3)$ & $(4)$ & $(5)$ & $(6)$ & $(7)$ & $(8)$ & $(9)$ & $(10)$ & $(11)$ & $(12)$ \\
\hline$V$ & $20.46 \pm 0.12$ & $2.51 \pm 0.05$ & 16.46 & 0.82 & 17.89 & 1.36 & 17.53 & 17.34 & $16.81 \pm 0.02$ & 16.83 & $0.60,1.05$ \\
$R$ & $20.14 \pm 0.10$ & $2.52 \pm 0.05$ & 16.13 & 0.76 & 18.34 & 1.46 & 17.1 & 16.97 & $16.68 \pm 0.02$ & 16.67 & $0.68,1.13$ \\
$I$ & $19.83 \pm 0.13$ & $2.50 \pm 0.06$ & 15.85 & 0.83 & 17.89 & 1.54 & 16.87 & 16.76 & $16.45 \pm 0.04$ & 16.48 & $0.69,1.13$ \\
\hline
\end{tabular}

Near the center, such an intensity distribution depends on the relative central intensity depression $q=\Delta I / I_{0}$, where $I_{0}$ is the central intensity obtained by extrapolation of the outer exponential slope with the scale length $\alpha$ to $R^{*}=0$, and $b \alpha$ is the cutoff-radius where the central flattening occurs.

Expressions (1) and (2) were fitted to the SBPs of the main body for radii $R^{*} \geq 9^{\prime \prime}$, where the colours become nearly constant (Fig. 4). Fits with a Sérsic profile give $\eta=$ $2.0 \pm 0.1$ and, as illustrated for the $V$ profile in Fig. 3a, are nearly indistinguishable from those using Eq. (2) with $b=3.0$ and $q=0.9$. The $V$ surface brightness distribution of the residual light in excess of the fit to the emission of the main body (Eq. (2)) is shown by small circles in the right panel of Fig. 3 .

Table 1 summarizes the derived photometric properties of the LSB host and the star-forming component of SBS $0940+544$. Columns 2 and 3 give respectively the central surface brightness $\mu_{\mathrm{E}, 0}$ and exponential scale length $\alpha^{\prime \prime}$ of the LSB host as obtained from linear fits to the SBPs for $R^{*} \geq 9^{\prime \prime}$ and weighted by the photometric uncertainty of each point. The corresponding integrated magnitude $m_{\mathrm{LSB}}^{\mathrm{fit}}$ of a pure exponential distribution $\mu_{\mathrm{E}, 0}-5 \log \left(\alpha^{\prime \prime}\right)-2$ is listed in Col. 4. As discussed above, the surface brightness distribution of the LSB host becomes significantly shallower for $R^{*}<9^{\prime \prime}$ than that predicted by inwards extrapolation of the exponential slope observed at the outskirts of SBS $0940+544$. Therefore, for the case under study $m_{\mathrm{LSB}}^{\mathrm{fit}}$ overestimates the apparent magnitude of the LSB host ( $\approx m_{\mathrm{LSB}}$; Col. 9 ). Columns 5 through 9 list quantities obtained from profile decomposition, with the intensity distribution of the LSB host modeled by Eq. (2). Columns 5 and 7 give respectively the radial extent $P_{25}$ and $E_{25}$ of the starburst and LSB components, determined at a surface brightness level of $25 \mathrm{mag} \operatorname{arcsec}^{-2}$ (cf. Fig. 3b). The apparent magnitudes of each component within $P_{25}$ and $E_{25}$ are listed in Cols. 6 and 8. Column 9 gives the apparent magnitude of the LSB component in 


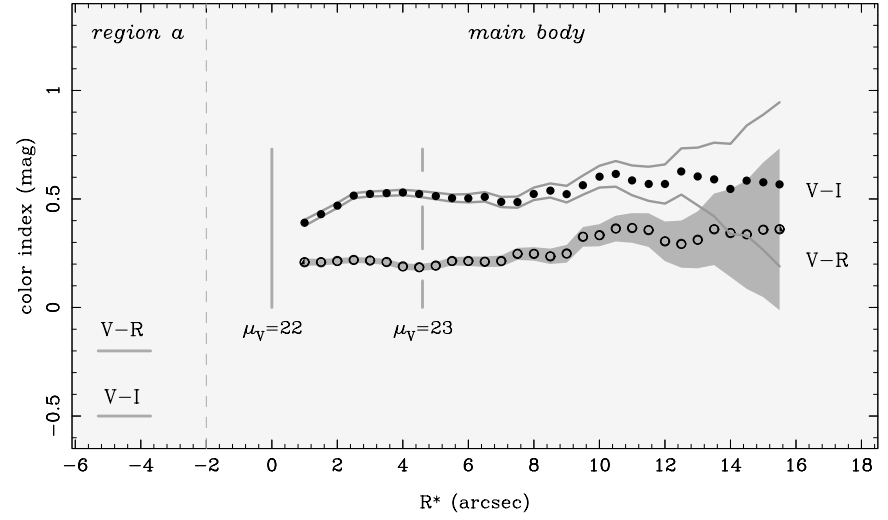

Fig. 4. $V-R$ and $V-I$ colour profiles of the main body of SBS $0940+544$ derived by subtraction of the surface brightness profiles in Fig. 3a. The radial range corresponding to the surface brightness interval between 22 and $23 \mathrm{~V}$ mag $\operatorname{arcsec}^{-2}$ is indicated by vertical lines. The average colours of the bright $\mathrm{H}$ II region $a$ (cf. Fig. 1a) $(V-R \sim-0.2 \mathrm{mag}$ and $V-I \lesssim$ $-0.5 \mathrm{mag})$ are indicated by horizontal lines.

each band within the photometric radius of $15^{\prime \prime} 5$, as derived from integration of the modeled distribution Eq. (2). The total magnitude of the BCD as inferred from SBP integration out to the same radius and by summing up the flux within a polygonal aperture is given in Cols. 10 and 11, respectively. Column 12 lists the effective radius $r_{\mathrm{eff}}$ and the radius $r_{80}$ which encircles $80 \%$ of the galaxy's total flux.

The $V-R$ and $V-I$ colour profiles of the LSB host of SBS $0940+544$ as derived by subtraction of the corresponding SBPs (Fig. 3a) are shown in Fig. 4. Both colour profiles show very weak gradients for $\mu_{V} \gtrsim$ $23 \mathrm{mag} \operatorname{arcsec}^{-2}$, and become nearly constant for $R^{*}>9^{\prime \prime}$. Beyond that radius the colour profiles (Fig. 4) give average colour indices $V-R=0.33 \pm 0.04 \mathrm{mag}$ and $V-I=$ $0.58 \pm 0.03 \mathrm{mag}$ for the LSB component. These values are consistent with the integrated colours $V-R=0.37 \mathrm{mag}$ and $V-I=0.58 \mathrm{mag}$, determined from the apparent magnitudes of the modeled intensity distribution of the LSB host within $R^{*}=15^{\prime \prime}$ (Table 1, Col. 9).

We have compared our results to those obtained for the $B$ and $R$ profiles by Doublier et al. (1997, 1999). The agreement is not satisfactory. The exponential scale length $\alpha$ of the LSB host derived here (Table 1 ) is very similar in all bands $\left(V, R\right.$ and $I$ ) being $\sim 2^{\prime \prime} .5(320 \mathrm{pc})$. Although Doublier et al. (1997) also report an exponential intensity decrease in $B$ and $R$ for radii $\gtrsim 3^{\prime \prime}$, they derive for the distance assumed here a $B$ and $R$ exponential scale length of $\sim 1 \mathrm{kpc}, 3$ times greater than our value. A striking disagreement is also evident from a comparison of the colour profiles derived here and those presented in Doublier et al. (1997). The $B-R$ colour profile of Doublier et al. (1997) shows a maximum of $\sim 0.5 \mathrm{mag}$ at $R^{*} \sim 0^{\prime \prime}$, then decreases monotonically with increasing radius to $\lesssim 0.2 \mathrm{mag}$. Our colour profile shows the opposite trend: very blue colours at small radii and nearly constant, moderately red $V-R$ and $V-I$ colours in the outskirts of SBS $0940+544$.

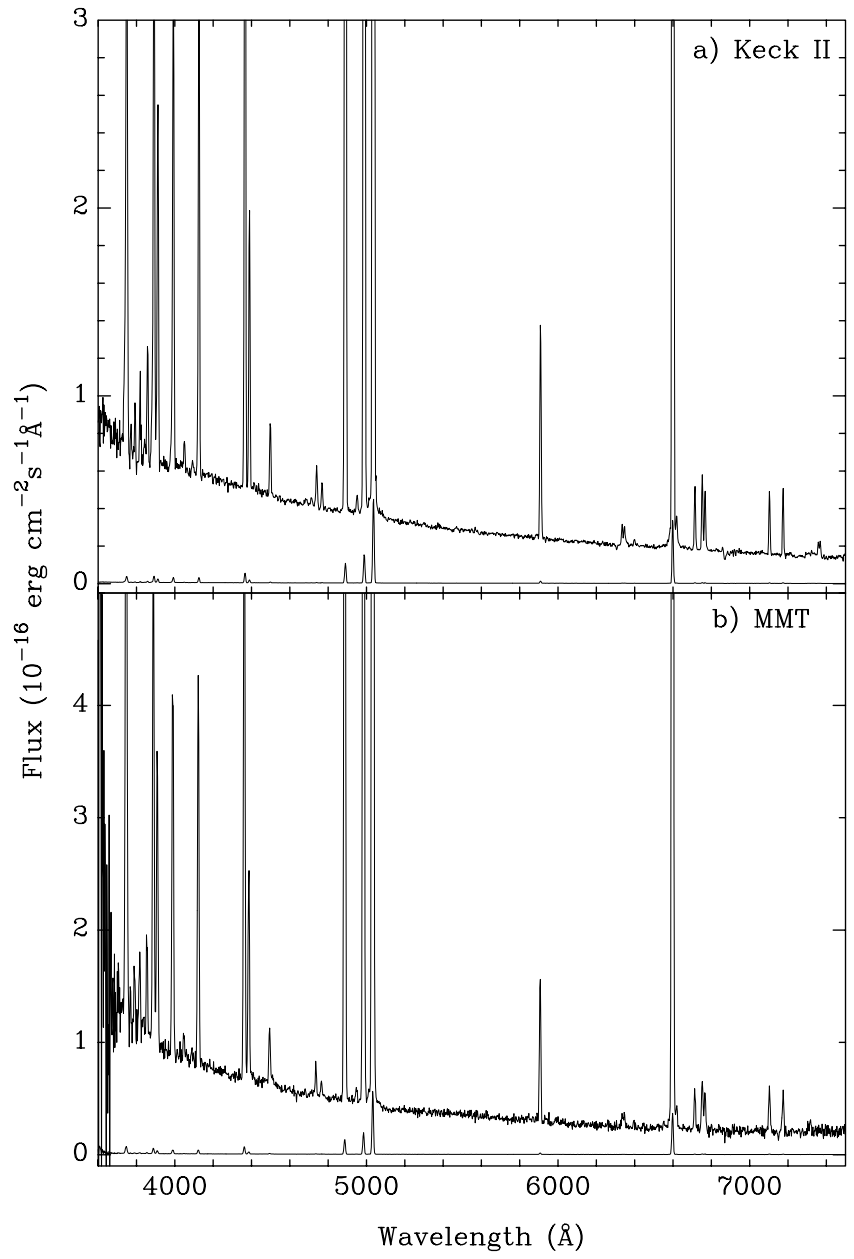

Fig. 5. Spectra of the brightest H II region $a$ in SBS 0940+544 obtained with: a) the Keck II telescope and b) the MMT. The lower spectra are the observed spectra downscaled by a factor of 100 .

\section{Chemical abundances}

Earlier determinations of element abundances in SBS 0940+544 (Izotov et al. 1991, 1994; Izotov \& Thuan 1998b) have revealed a metallicity ranging from $Z_{\odot} / 31$ to $Z_{\odot} / 25$, among the lowest for BCDs. Abundance determinations in this galaxy are therefore important for studying the origin of elements in low-metallicity environments and deriving the primordial helium abundance. We emphasize that repeated observations of the same galaxy are very useful to estimate the robustness of the abundance determinations. This was one of the motivations for carrying out new MMT and Keck II observations of SBS $0940+544$ and analyzing the abundance pattern in its brightest $\mathrm{H}$ II region. The spectra of region $a$ used for the abundance determination are shown in Fig. 5.

A two-zone photoionized $\mathrm{H}$ II region has been assumed for abundance determination; the electron temperature $T_{\mathrm{e}}(\mathrm{O}$ III $)$ for the high-ionization region has been derived from the observed flux ratio [O III] $\lambda 4363 /(\lambda 4959+5007)$, using a five-level atom model (Aller 1984) with atomic data from Mendoza (1983). The electron temperature $T_{\mathrm{e}}(\mathrm{O}$ II) for the low-ionization region has been derived 
using the empirical relation between $T_{\mathrm{e}}(\mathrm{O}$ II $)$ and $T_{\mathrm{e}}(\mathrm{O}$ III $)$ from the H II region photoionization models by Stasińska (1990). The $[\mathrm{S}$ II $] \lambda 6717 / \lambda 6731$ ratio was used to derive the electron number density $N_{\mathrm{e}}(\mathrm{S}$ II).

The spectra were corrected for interstellar extinction, where the extinction coefficient $C(\mathrm{H} \beta)$ was derived from the hydrogen Balmer decrement using the equations given in Izotov et al. (1994) and the theoretical hydrogen emission line flux ratios from Brocklehurst (1971).

The emission line fluxes were measured using a Gaussian profile fitting. The errors of the line fluxes measurements include the errors in the fitting of profiles and those in the placement of the continuum. We also take into account the errors introduced by uncertainties in the spectral energy distributions of standard stars. Standard star flux deviations for both standard stars Feige 34 and HZ 44 are taken to be 1\% (Oke 1990; Bohlin 1996). These errors have been propagated in the calculations of the element abundance errors. The observed $(F(\lambda))$ and extinctioncorrected $(I(\lambda))$ emission line fluxes relative to the $\mathrm{H} \beta$ emission line fluxes, the equivalent widths $E W$ of the emission lines, the extinction coefficient $C(\mathrm{H} \beta)$, the observed flux of the $\mathrm{H} \beta$ emission line, and the equivalent width of the hydrogen absorption lines for region $a$, derived from Keck II and MMT spectra with the slit position $\mathrm{PA}=-41^{\circ}$ are listed in Table 2 . The derived extinction coefficient $C(\mathrm{H} \beta)$ is small.

The ionic abundances of $\mathrm{O}^{+2}, \mathrm{Ne}^{+2}, \mathrm{Ar}^{+3}, \mathrm{He}^{+}$ and $\mathrm{He}^{+2}$ were obtained using the electron temperature $T_{\mathrm{e}}(\mathrm{O}$ III $)$. The electron temperature $T_{\mathrm{e}}(\mathrm{O}$ II $)$ is adopted to calculate the $\mathrm{O}^{+}, \mathrm{N}^{+}, \mathrm{S}^{+}$and $\mathrm{Fe}^{+2}$ ionic abundances, while the intermediate value of the electron temperature $T_{\mathrm{e}}\left(\mathrm{S}\right.$ III) was used to derive the ionic abundances of $\mathrm{Ar}^{+2}$ and $\mathrm{S}^{+2}$ (Garnett 1992).

The total heavy element abundances were obtained using ionization correction factors (ICF) following Izotov et al. (1994, 1997c) and Thuan et al. (1995). For oxygen the total abundance is the sum of $\mathrm{O}^{+}, \mathrm{O}^{+2}$ and $\mathrm{O}^{+3}$ ion abundances. Although emission lines of $\mathrm{O}^{+3}$ are absent in the optical spectrum, this ion resides in the $\mathrm{He}^{+2}$ region. Therefore, we derive the $\mathrm{O}^{+3}$ ion abundance using the He II $\lambda 4686 \AA$ emission line intensity as described by Izotov \& Thuan (1999). The ionic and heavy element abundances for the brightest H II region in SBS 0940+544 together with electron temperatures and electron number densities are given in Table 3 along with the adopted ionization correction factors.

The oxygen abundance derived from the Keck II and MMT spectra are $12+\log (\mathrm{O} / \mathrm{H})=7.50 \pm 0.01$ and $7.46 \pm$ 0.02 respectively. For comparison, Izotov et al. (1991) derived $12+\log (\mathrm{O} / \mathrm{H})=7.52 \pm 0.12$, while Izotov et al. (1994) obtained $12+\log (\mathrm{O} / \mathrm{H})=7.37 \pm 0.02$. The former value was calculated using a three-level atom model. If the five-level atom model is adopted instead then the oxygen abundance is decreased by $\sim 0.04$ dex (Izotov \& Thuan 1999). The latter value is significantly lower than the previous ones. However, Izotov \& Thuan (1998b) noted that Izotov et al. (1994) used an erroneously low [O III] $\lambda 5007$ emission line intensity. They rederived the oxygen abundance using the data of Izotov et al. (1994) and found $12+$ $\log (\mathrm{O} / \mathrm{H})=7.43 \pm 0.01$. Thus, all $12+\log (\mathrm{O} / \mathrm{H})$ values are confined in the narrow range $7.43-7.50$, or $Z_{\odot} / 31-$ $Z_{\odot} / 26 .^{3}$

The ratios of other heavy element abundances to the oxygen abundance derived from the Keck II and MMT observations (Table 3) are mutually consistent and they are also in agreement with the heavy element abundance ratios derived earlier in SBS $0940+544$ and in other lowmetallicity BCDs (Izotov \& Thuan 1999).

The very low metallicity of SBS $0940+544$ and the presence of very strong emission lines in its spectrum makes it one of the best galaxies for the determination of the primordial helium abundance. For this, we use the five strongest He I $\lambda 3889, \lambda 4471, \lambda 5876, \lambda 6678$ and $\lambda 7065$ emission lines in both Keck II and MMT spectra. The first and last He I emission lines are more sensitive to collisional and fluorescent enhancement mechanisms. They are used to correct other He I emission lines for these effects (Izotov et al. 1994, 1997c). The helium abundance is derived from the corrected intensities of the He I $\lambda 4471, \lambda 5876, \lambda 6678$ emission lines and is shown in Table 3 . The mean values of the ${ }^{4} \mathrm{He}$ mass fraction $Y=0.247 \pm 0.003$ (Keck II) and $Y=0.244 \pm 0.006$ (MMT) (see Table 3 ) are consistent with the previously derived value $Y=0.247 \pm 0.007$ (Izotov \& Thuan 1998b) and they are close to the primordial ${ }^{4} \mathrm{He}$ mass fraction $Y_{\mathrm{p}}=0.244 \pm 0.002$ derived by extrapolating the $Y$ vs. $\mathrm{O} / \mathrm{H}$ linear regression to $\mathrm{O} / \mathrm{H}=0$ (Izotov \& Thuan 1998b), or to $Y_{\mathrm{p}}=0.245 \pm 0.002$ derived in the two most metal-deficient BCDs I Zw 18 and SBS 0335-052 (Izotov et al. 1999).

It was pointed out in Sect. 2.2 that atmospheric dispersion may introduce some uncertainties in the abundance determination. To estimate these uncertainties we measure the fluxes of the [O $\mathrm{II}] \lambda 3727$ and [O III] $\lambda 4363$ emission lines in an aperture $1^{\prime \prime} \times 1^{\prime \prime}$ placed on the maximum in the flux distribution, and those in an aperture with the same size but displaced along the slit by $00^{\prime \prime} 4$ and 0 "' 1 respectively for the [O II] and [O III] lines. We obtain relative flux differences of $\sim 7 \%$ and $\sim 1 \%$ for the $[\mathrm{O}$ II] and $[\mathrm{O}$ III] emission lines. The effect of the atmospheric dispersion results in an uncertainty not exceeding $1 \%$ of the total oxygen abundance, because oxygen in the $\mathrm{H}$ II region is mainly $\mathrm{O}^{+2}$ (see Table 3 ). However, because the $\mathrm{H}$ II region is much more extended than the size of the aperture used for analysis of the atmospheric dispersion, and of the large apertures used in the extraction of spectra for the abundance analysis, the effect of atmospheric dispersion is expected to be smaller for the [O II] $\lambda 3727$ emission line and negligible compared to the statistical errors for the $[\mathrm{O}$ III $] \lambda 4363$ emission line. Therefore, we decided not to include it in the error budget.

\footnotetext{
${ }^{3} 12+\log (\mathrm{O} / \mathrm{H})_{\odot}=8.92$ (Anders \& Grevesse 1989).
} 
Table 2. Observed $(F(\lambda))$, extinction-corrected $(I(\lambda))$ fluxes and equivalent widths $(E W)$ of the emission lines in the spectra of the brightest $\mathrm{H}$ II region in SBS $0940+544$.

\begin{tabular}{|c|c|c|c|c|c|c|}
\hline \multirow[b]{2}{*}{$\lambda_{0}(\AA)$ Ion } & \multicolumn{3}{|c|}{ Keck II } & \multicolumn{3}{|c|}{ MMT } \\
\hline & $F(\lambda) / F(\mathrm{H} \beta)$ & $I(\lambda) / I(\mathrm{H} \beta)$ & $E W(\AA)$ & $F(\lambda) / F(\mathrm{H} \beta)$ & $I(\lambda) / I(\mathrm{H} \beta)$ & $E W(\AA)$ \\
\hline $3727[\mathrm{O}$ II] & $0.445 \pm 0.007$ & $0.471 \pm 0.008$ & $62.0 \pm 0.3$ & $0.585 \pm 0.008$ & $0.587 \pm 0.008$ & $62.7 \pm 0.7$ \\
\hline 3750 H12 & $0.013 \pm 0.001$ & $0.022 \pm 0.002$ & $1.9 \pm 0.2$ & $0.022 \pm 0.003$ & $0.023 \pm 0.004$ & $2.5 \pm 0.3$ \\
\hline 3771 H11 & $0.023 \pm 0.001$ & $0.032 \pm 0.002$ & $3.3 \pm 0.2$ & $0.044 \pm 0.003$ & $0.045 \pm 0.005$ & $4.9 \pm 0.4$ \\
\hline 3798 H10 & $0.042 \pm 0.001$ & $0.052 \pm 0.002$ & $6.1 \pm 0.2$ & $0.059 \pm 0.004$ & $0.060 \pm 0.005$ & $6.7 \pm 0.5$ \\
\hline 3820 [He I] & $0.017 \pm 0.001$ & $0.018 \pm 0.001$ & $2.5 \pm 0.3$ & $\ldots$ & $\ldots$ & 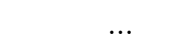 \\
\hline 3835 H9 & $0.058 \pm 0.001$ & $0.069 \pm 0.002$ & $8.6 \pm 0.2$ & $0.064 \pm 0.004$ & $0.065 \pm 0.005$ & $7.4 \pm 0.4$ \\
\hline 3868 [Ne III] & $0.340 \pm 0.005$ & $0.356 \pm 0.006$ & $49.6 \pm 0.3$ & $0.373 \pm 0.006$ & $0.374 \pm 0.006$ & $44.0 \pm 0.7$ \\
\hline $3889 \mathrm{H} 8+\mathrm{He} \mathrm{I}$ & $0.184 \pm 0.003$ & $0.201 \pm 0.004$ & $26.9 \pm 0.3$ & $0.204 \pm 0.005$ & $0.205 \pm 0.006$ & $25.1 \pm 0.6$ \\
\hline $3968[\mathrm{Ne}$ III $]+\mathrm{H} 7$ & $0.259 \pm 0.004$ & $0.278 \pm 0.005$ & $38.3 \pm 0.3$ & $0.288 \pm 0.005$ & $0.290 \pm 0.006$ & $39.8 \pm 0.7$ \\
\hline 4026 He I & $0.014 \pm 0.001$ & $0.014 \pm 0.001$ & $2.1 \pm 0.2$ & $0.019 \pm 0.004$ & $0.019 \pm 0.004$ & $2.7 \pm 0.5$ \\
\hline 4068 [S II] & $0.009 \pm 0.001$ & $0.009 \pm 0.001$ & $1.4 \pm 0.2$ & $0.007 \pm 0.003$ & $0.007 \pm 0.003$ & $1.0 \pm 0.4$ \\
\hline $4101 \mathrm{H} \delta$ & $0.244 \pm 0.004$ & $0.259 \pm 0.004$ & $39.3 \pm 0.3$ & $0.267 \pm 0.005$ & $0.269 \pm 0.006$ & $40.0 \pm 0.7$ \\
\hline $4340 \mathrm{H} \gamma$ & $0.461 \pm 0.007$ & $0.477 \pm 0.007$ & $84.9 \pm 0.4$ & $0.487 \pm 0.007$ & $0.488 \pm 0.007$ & $86.9 \pm 1.0$ \\
\hline 4363 [O III] & $0.125 \pm 0.002$ & $0.128 \pm 0.002$ & $23.2 \pm 0.3$ & $0.142 \pm 0.004$ & $0.142 \pm 0.004$ & $27.0 \pm 0.7$ \\
\hline 4388 Не I & $0.003 \pm 0.001$ & $0.003 \pm 0.001$ & $0.6 \pm 0.2$ & $\ldots$ & $\ldots$ & $\ldots$ \\
\hline $4471 \mathrm{He} \mathrm{I}$ & $0.036 \pm 0.001$ & $0.037 \pm 0.001$ & $7.4 \pm 0.2$ & $0.039 \pm 0.003$ & $0.039 \pm 0.003$ & $7.3 \pm 0.6$ \\
\hline 4658 [Fe III] & $0.005 \pm 0.001$ & $0.005 \pm 0.001$ & $1.2 \pm 0.3$ & $0.003 \pm 0.002$ & $0.003 \pm 0.002$ & $0.7 \pm 0.5$ \\
\hline 4686 Не II & $0.006 \pm 0.001$ & $0.006 \pm 0.001$ & $1.3 \pm 0.3$ & $\ldots$ & $\ldots$ & $\ldots$ \\
\hline $4711[$ Ar IV $]+$ He I & $0.022 \pm 0.001$ & $0.022 \pm 0.001$ & $5.2 \pm 0.3$ & $0.020 \pm 0.002$ & $0.020 \pm 0.002$ & $4.6 \pm 0.5$ \\
\hline $4740[\mathrm{Ar}$ IV] & $0.013 \pm 0.001$ & $0.013 \pm 0.001$ & $3.0 \pm 0.2$ & $0.012 \pm 0.003$ & $0.012 \pm 0.003$ & $2.9 \pm 0.6$ \\
\hline $4861 \mathrm{H} \beta$ & $1.000 \pm 0.015$ & $1.000 \pm 0.015$ & $241.1 \pm 0.8$ & $1.000 \pm 0.011$ & $1.000 \pm 0.011$ & $250.1 \pm 1.9$ \\
\hline $4922 \mathrm{He} \mathrm{I}$ & $0.010 \pm 0.001$ & $0.010 \pm 0.001$ & $2.4 \pm 0.3$ & $0.010 \pm 0.002$ & $0.010 \pm 0.002$ & $2.6 \pm 0.6$ \\
\hline 4959 [O III] & $1.415 \pm 0.021$ & $1.402 \pm 0.021$ & $348.8 \pm 1.0$ & $1.436 \pm 0.014$ & $1.435 \pm 0.014$ & $381.3 \pm 2.3$ \\
\hline 5007 [O III $]$ & $4.251 \pm 0.061$ & $4.204 \pm 0.061$ & $1052.0 \pm 1.7$ & $4.218 \pm 0.034$ & $4.215 \pm 0.034$ & $1154.2 \pm 3.9$ \\
\hline $5199[\mathrm{~N} \mathrm{I}]$ & $0.003 \pm 0.001$ & $0.003 \pm 0.001$ & $0.9 \pm 0.2$ & $\ldots$ & $\ldots$ & $\ldots$ \\
\hline 5271 [Fe III] & $0.004 \pm 0.001$ & $0.004 \pm 0.001$ & $1.2 \pm 0.3$ & $\ldots$ & $\ldots$ & $\ldots$ \\
\hline 5876 He I & $0.102 \pm 0.002$ & $0.097 \pm 0.002$ & $39.0 \pm 0.5$ & $0.097 \pm 0.003$ & $0.097 \pm 0.003$ & $39.2 \pm 1.1$ \\
\hline $6300[\mathrm{O}$ I] & $0.011 \pm 0.001$ & $0.010 \pm 0.001$ & $4.9 \pm 0.4$ & $0.007 \pm 0.002$ & $0.007 \pm 0.002$ & $3.4 \pm 0.7$ \\
\hline 6312 [S III] & $0.012 \pm 0.001$ & $0.011 \pm 0.001$ & $5.3 \pm 0.3$ & $0.010 \pm 0.002$ & $0.010 \pm 0.002$ & $4.7 \pm 0.9$ \\
\hline $6363[\mathrm{O}$ I $]$ & $0.004 \pm 0.001$ & $0.004 \pm 0.001$ & $1.9 \pm 0.3$ & & $\ldots$ & \\
\hline $6563 \mathrm{H} \alpha$ & $2.946 \pm 0.043$ & $2.750 \pm 0.043$ & $1409.0 \pm 2.5$ & $2.761 \pm 0.024$ & $2.733 \pm 0.025$ & $1438.9 \pm 6.0$ \\
\hline 6584 [N II] & $0.013 \pm 0.001$ & $0.012 \pm 0.001$ & $3.9 \pm 0.3$ & $0.016 \pm 0.002$ & $0.016 \pm 0.002$ & $8.6 \pm 1.1$ \\
\hline $6678 \mathrm{He} \mathrm{I}$ & $0.029 \pm 0.001$ & $0.027 \pm 0.001$ & $14.9 \pm 0.5$ & $0.027 \pm 0.002$ & $0.027 \pm 0.002$ & $14.3 \pm 1.0$ \\
\hline 6717 [S II] & $0.038 \pm 0.001$ & $0.035 \pm 0.001$ & $19.2 \pm 0.4$ & $0.038 \pm 0.002$ & $0.038 \pm 0.002$ & $20.9 \pm 1.3$ \\
\hline 6731 [S II] & $0.030 \pm 0.001$ & $0.028 \pm 0.001$ & $15.3 \pm 0.4$ & $0.026 \pm 0.002$ & $0.026 \pm 0.002$ & $14.7 \pm 1.1$ \\
\hline $7065 \mathrm{He} \mathrm{I}$ & $0.026 \pm 0.001$ & $0.024 \pm 0.001$ & $14.7 \pm 0.4$ & $0.032 \pm 0.002$ & $0.032 \pm 0.002$ & $19.8 \pm 1.2$ \\
\hline 7136 [Ar III] & $0.028 \pm 0.001$ & $0.026 \pm 0.001$ & $16.5 \pm 0.5$ & $0.029 \pm 0.002$ & $0.029 \pm 0.002$ & $17.9 \pm 1.2$ \\
\hline 7320 [O II] & $0.007 \pm 0.001$ & $0.007 \pm 0.001$ & $4.6 \pm 0.6$ & $\ldots$ & $\ldots$ & $\ldots$ \\
\hline 7330 [O II] & $0.007 \pm 0.001$ & $0.006 \pm 0.001$ & $4.1 \pm 0.4$ & $\ldots$ & $\ldots$ & $\ldots$ \\
\hline$C(\mathrm{H} \beta) \operatorname{dex}$ & & $0.085 \pm 0.019$ & & & $0.005 \pm 0.011$ & \\
\hline$F(\mathrm{H} \beta)^{a}$ & & $1.21 \pm 0.01$ & & & $1.22 \pm 0.01$ & \\
\hline$E W(\mathrm{abs}) \AA$ & & $1.1 \pm 0.1$ & & & $0.1 \pm 0.3$ & \\
\hline
\end{tabular}

${ }^{a}$ In units $10^{-14} \mathrm{erg} \mathrm{s}^{-1} \mathrm{~cm}^{-2}$.

\section{Age of the underlying stellar population}

One of the key problems debated in recent years is the evolutionary status of very metal-deficient BCDs: are these galaxies young or old? To resolve this problem an analysis of the stellar populations is required. The metallicity of the galaxy is one of the key parameters that determines the photometric properties of stellar populations. Izotov \& Thuan (1999), considering the abundance ratios of the heavy elements in BCDs, have suggested that the BCDs with $12+\log (\mathrm{O} / \mathrm{H}) \lesssim 7.6$ might be young unevolved galaxies. SBS $0940+544$ fulfills this abundance condition. Furthermore, the nitrogen-to-oxygen abundance ratios $\log (\mathrm{N} / \mathrm{O})=-1.65 \pm 0.02$ and $-1.60 \pm 0.05$ derived respectively from the Keck II and MMT spectra (Table 3) are confined to the narrow range obtained earlier by Thuan et al. (1995) and Izotov \& Thuan (1999) for extremely metal-poor BCDs. The constancy of the 
Table 3. Physical conditions and element abundances in the brightest H II region of SBS $0940+544$.

\begin{tabular}{|c|c|c|}
\hline Value & Keck II & $\overline{\mathrm{MMT}}$ \\
\hline$\overline{T_{\mathrm{e}}(\mathrm{O} \text { III })(\mathrm{K})}$ & $18830 \pm 220$ & $20000 \pm 370$ \\
\hline$T_{\mathrm{e}}(\mathrm{O}$ II $)(\mathrm{K})$ & $15320 \pm 170$ & $15690 \pm 260$ \\
\hline$T_{\mathrm{e}}(\mathrm{S}$ III $)(\mathrm{K})$ & $17330 \pm 190$ & $18300 \pm 300$ \\
\hline$N_{\mathrm{e}}(\mathrm{S}$ II $)\left(\mathrm{cm}^{-3}\right)$ & $170 \pm 60$ & $10 \pm 10$ \\
\hline$N_{\mathrm{e}}($ He II $)\left(\mathrm{cm}^{-3}\right)$ & $10 \pm 10$ & $60 \pm 10$ \\
\hline$\tau(\lambda 3889)$ & 0.0 & $0.5 \pm 0.3$ \\
\hline $\mathrm{O}^{+} / \mathrm{H}^{+}\left(\times 10^{5}\right)$ & $0.389 \pm 0.013$ & $0.447 \pm 0.020$ \\
\hline $\mathrm{O}^{+2} / \mathrm{H}^{+}\left(\times 10^{5}\right)$ & $2.727 \pm 0.081$ & $2.438 \pm 0.102$ \\
\hline $\mathrm{O}^{+3} / \mathrm{H}^{+}\left(\times 10^{6}\right)$ & $0.201 \pm 0.033$ & the- \\
\hline $\mathrm{O} / \mathrm{H}\left(\times 10^{5}\right)$ & $3.137 \pm 0.082$ & $2.885 \pm 0.116$ \\
\hline $12+\log (\mathrm{O} / \mathrm{H})$ & $7.497 \pm 0.011$ & $7.460 \pm 0.018$ \\
\hline $\mathrm{N}^{+} / \mathrm{H}^{+}\left(\times 10^{7}\right)$ & $0.874 \pm 0.034$ & $1.115 \pm 0.112$ \\
\hline $\mathrm{ICF}(\mathrm{N})^{a}$ & 8.05 & 6.46 \\
\hline $\log (\mathrm{N} / \mathrm{O})$ & $-1.649 \pm 0.021$ & $-1.603 \pm 0.048$ \\
\hline $\mathrm{Ne}^{+2} / \mathrm{H}^{+}\left(\times 10^{5}\right)$ & $0.476 \pm 0.015$ & $0.436 \pm 0.020$ \\
\hline $\operatorname{ICF}(\mathrm{Ne})^{a}$ & 1.15 & 1.18 \\
\hline $\log (\mathrm{Ne} / \mathrm{O})$ & $-0.758 \pm 0.018$ & $-0.748 \pm 0.028$ \\
\hline $\mathrm{S}^{+} / \mathrm{H}^{+}\left(\times 10^{7}\right)$ & $0.608 \pm 0.015$ & $0.583 \pm 0.031$ \\
\hline $\mathrm{S}^{+2} / \mathrm{H}^{+}\left(\times 10^{7}\right)$ & $3.643 \pm 0.213$ & $2.794 \pm 0.516$ \\
\hline $\operatorname{ICF}(\mathrm{S})^{a}$ & 2.10 & 1.83 \\
\hline $\log (\mathrm{S} / \mathrm{O})$ & $-1.546 \pm 0.025$ & $-1.670 \pm 0.069$ \\
\hline $\mathrm{Ar}^{+2} / \mathrm{H}^{+}\left(\times 10^{7}\right)$ & $0.744 \pm 0.021$ & $0.751 \pm 0.050$ \\
\hline $\mathrm{Ar}^{+3} / \mathrm{H}^{+}\left(\times 10^{7}\right)$ & $1.124 \pm 0.069$ & $0.959 \pm 0.205$ \\
\hline $\operatorname{ICF}(\mathrm{Ar})^{a}$ & 1.01 & 1.02 \\
\hline $\log (\mathrm{Ar} / \mathrm{O})$ & $-2.219 \pm 0.020$ & $-2.219 \pm 0.059$ \\
\hline $\mathrm{Fe}^{+2} / \mathrm{H}^{+}\left(\times 10^{7}\right)$ & $0.934 \pm 0.212$ & $0.547 \pm 0.384$ \\
\hline $\mathrm{ICF}(\mathrm{Fe})^{a}$ & 10.1 & 8.07 \\
\hline $\log (\mathrm{Fe} / \mathrm{O})$ & $-1.537 \pm 0.099$ & $-1.815 \pm 0.313$ \\
\hline$[\mathrm{O} / \mathrm{Fe}]$ & $0.103 \pm 0.099$ & $0.395 \pm 0.313$ \\
\hline $\mathrm{He}^{+} / \mathrm{H}^{+}(\lambda 4471)$ & $0.0801 \pm 0.0023$ & $0.0829 \pm 0.0066$ \\
\hline $\mathrm{He}^{+} / \mathrm{H}^{+}(\lambda 5876)$ & $0.0821 \pm 0.0015$ & $0.0805 \pm 0.0024$ \\
\hline $\mathrm{He}^{+} / \mathrm{H}^{+}(\lambda 6678)$ & $0.0813 \pm 0.0023$ & $0.0804 \pm 0.0055$ \\
\hline $\mathrm{He}^{+} / \mathrm{H}^{+}($mean $)$ & $0.0815 \pm 0.0011$ & $0.0807 \pm 0.0021$ \\
\hline $\mathrm{He}^{+2} / \mathrm{H}^{+}(\lambda 4686)$ & $0.0005 \pm 0.0001$ & $\ldots$ \\
\hline $\mathrm{He} / \mathrm{H}$ & $0.0820 \pm 0.0011$ & $0.0807 \pm 0.0021$ \\
\hline$Y$ & $0.2468 \pm 0.0034$ & $0.2439 \pm 0.0066$ \\
\hline
\end{tabular}

${ }^{a} \mathrm{ICF}$ is the ionization correction factor.

$\mathrm{N} / \mathrm{O}$ abundance ratio in these BCDs with a very small spread of values around the mean suggests that the production of primary nitrogen occurs in massive stars only and hence these systems are likely to be young, since intermediate-mass stars have not had time to release their nucleosynthetic products. On the other hand, some recent chemical evolution models have been succesful in reproducing the observed constancy of the $\mathrm{N} / \mathrm{O}$ ratio, with nitrogen produced by the longer-lived intermediate-mass stars (Pilyugin 1999; Henry et al. 2000).

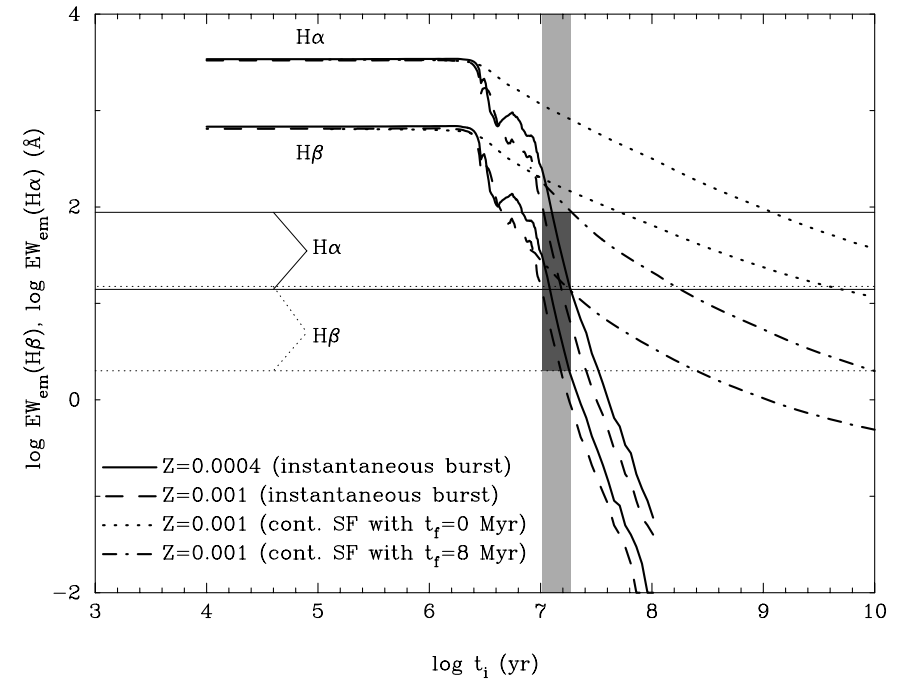

Fig. 6. Equivalent widths of the nebular emission lines $\mathrm{H} \alpha$ and $\mathrm{H} \beta$ vs. age for an instantaneous burst and a heavy element mass fraction $Z=1 / 20 Z_{\odot}$ (thick dashed lines) and $Z=$ $1 / 50 Z \odot$ (thick solid lines) calculated with the PEGASE. 2 code (Fioc \& Rocca-Volmerange 1997). Model predictions are also shown for the case of constant continuous star formation starting at an age defined by the abscissa $t_{\mathrm{i}}$ and stopping at $t_{\mathrm{f}}$, with $t_{\mathrm{f}}=0 \mathrm{Myr}$ (dotted lines), and $t_{\mathrm{f}}=8 \mathrm{Myr}$ (dash-dotted lines). Thin solid and dotted horizontal lines show the upper and lower limits of observed $E W(\mathrm{H} \alpha)$ and $E W(\mathrm{H} \beta)$ for 4 regions of the underlying galaxy. The shaded rectangular region shows the age limits in the case of an instantaneous burst.

Deep $V, R, I$, and $\mathrm{H} \alpha$ imaging, high signal-to-noise ratio spectra showing strong emission lines and the detection of hydrogen Balmer absorption lines in the main body of SBS 0940+544 enable the study of stellar populations and constraint of their ages through various techniques.

The light of the brightest $\mathrm{H}$ II region of the galaxy is dominated by a very young stellar population. The very blue continuum and large $E W(\mathrm{H} \beta)=241-250 \AA$ (Fig. 5, Table 2) are consistent with an age of an instantaneous burst not exceeding $4 \mathrm{Myr}$, for a metallicity $Z=1 / 20$ $1 / 50 Z_{\odot}$ (Fig. 6). However, despite that young age, no Wolf-Rayet stars are detected in our high signal-to-noise ratio spectra. We also note that the nebular He II $\lambda 4686$ emission line is not detected in the MMT spectrum and its intensity in the Keck II spectrum is only $0.6 \%$ of $\mathrm{H} \beta$, or several times weaker than in the very metal-deficient BCDs I Zw 18, SBS 0335-052 and Tol 1214-277 with detected Wolf-Rayet stellar populations (Izotov et al. 1997a; Izotov et al. 1999; Fricke et al. 2001).

The interpretation of the photometric data for the brightest $\mathrm{H}$ II region is not straightforward because of the strong contamination of stellar radiation by the emission of the ionized gas. The colour $V-I \sim-0.5 \mathrm{mag}$ is too blue to be explained even by the youngest stellar population, because $\sim 40 \%$ of the $V$ light is contributed by the very strong oxygen emission lines [O III] $\lambda 4959, \lambda 5007$. Therefore broad-band photometry alone is not sufficient to constrain the age of young star-forming regions. However, 
spectroscopic data in conjunction with photometric data allows us to disentangle stellar from gaseous emission.

Data for bright $\mathrm{H}$ II regions do not permit the detection of the small fraction of light contributed by an old stellar population (e.g., Papaderos et al. 1998; Fricke et al. 2001; Noeske et al. 2000). The old population in such regions, if present, is hidden by the strong emission from the young stars and the gas ionized by the numerous massive stars in the star-forming region. More promising for the detection of an old stellar population is the study of the underlying extended emission from the host galaxy as gaseous contamination is minimized there. Nearly all BCDs show an underlying stellar component with red colours, consistent with ages greater than a few Gyr (Loose \& Thuan 1986; Papaderos et al. 1996a, 1996b; Telles \& Terlevich 1997). However, the colours of the extended emission in the few very low metallicity BCDs are consistent with younger ages.

Another method for deriving ages is by fitting the observed galaxy spectral energy distribution (SED) with theoretical SEDs for various stellar population ages and star formation histories. This method has been applied to several extremely metal-deficient BCDs with $Z=(1 / 20-$ $1 / 40) Z_{\odot}$ (e.g., SBS 0335-052, Papaderos et al. 1998; SBS 1415+437, Thuan et al. 1999a; and Tol 1214-277, Fricke et al. 2001). The SED of the underlying stellar component in these galaxies, obtained after subtraction of the ionized gas emission from the observed spectrum, could be fitted by a stellar population not older than a few hundred Myr.

However, both methods are subject to uncertainties introduced by extinction. Therefore, other less extinctiondependent methods are desirable to better constrain the age of stellar populations. We use in this section two such methods. One relies on the Balmer nebular emission line equivalent widths and the other on the Balmer stellar absorption line equivalent widths. Such an analysis is feasible because of the exceptionally high quality of the Keck II and MMT spectra. Because age estimates from all the above mentioned methods depend on the adopted star formation history in the galaxy, we next consider different scenarios of star formation (instantaneous burst, continuous star formation) with varying extinction and star formation rate (SFR). We then put together the constraints imposed on the stellar age and extinction by all four methods to draw a consistent picture for the populations in SBS $0940+544$.

\subsection{The case of an instantaneous burst}

\subsubsection{Age from the nebular emission lines}

Under the assumption of an ionization-bounded H II region, the strongest hydrogen recombination emission lines $\mathrm{H} \alpha$ and $\mathrm{H} \beta$ provide an estimate of the age of the young stellar population when late $\mathrm{O}$ and early $\mathrm{B}$ stars are still present. The ionizing flux from such a young stellar population and hence the equivalent widths of the Balmer emission lines have a very strong temporal evolution.
Fluxes and equivalent widths of the $\mathrm{H} \alpha$ and $\mathrm{H} \beta$ emission lines are obtained for all four regions in the main body. Because the $\mathrm{H} \beta$ emission line is narrower than the absorption line in these regions and does not fill the absorption component, its flux was measured using the continuum level at the bottom of the absorption line. This level has been chosen by visually interpolating from the absorption line wings to the center of the line. The extinction coefficient $C(\mathrm{H} \beta)$ in those regions is derived from the $\mathrm{H} \alpha / \mathrm{H} \beta$ flux ratio. For this, the theoretical recombination $\mathrm{H} \alpha / \mathrm{H} \beta$ flux ratio of 2.8 is adopted, which is typical for hot low-metallicity H II regions. No correction for the absorption line equivalent width has been made in this case. Results of the measurements together with errors are shown in Table 4. In Fig. 6 we compare the measured $\mathrm{H} \alpha$ and $\mathrm{H} \beta$ emission line equivalent widths with those predicted at a given age of an instantaneous burst. The time evolution of the $\mathrm{H} \alpha$ and $\mathrm{H} \beta$ emission line equivalent widths for the heavy element mass fractions $Z_{\odot} / 50$ (thick solid lines) and $Z_{\odot} / 20$ (thick dashed lines) is calculated using the galactic evolution code PEGASE.2 (Fioc \& Rocca-Volmerange 1997). This code is based on the Padua isochrones (Bertelli et al. 1994) and stellar atmosphere models from Lejeune et al. (1998). Thin solid and dotted horizontal lines indicate respectively the highest and lowest observed equivalent widths of $\mathrm{H} \alpha$ and $\mathrm{H} \beta$ for the 4 regions of the underlying galaxy. The shaded region shows that the ages range between 10 and $20 \mathrm{Myr}$, meaning that the gas in the main body is likely ionized by early $\mathrm{B}$ stars rather than by $\mathrm{O}$ stars.

This age estimate is valid if the population of ionizing stars is large enough and can be described by an initial mass function (IMF) with an analytical form, for example a Salpeter IMF. However, in the case of the main body of SBS 0940+544, the number of ionizing stars is small and stochastic effects in the distribution of stars of different masses might be important. In particular, because of stochastic star formation, the ionization may be caused by a few $\mathrm{O}$ stars instead of a population of $\mathrm{B}$ stars. Taking the number of ionizing Lyc photons to be respectively $10^{49} \mathrm{~s}^{-1}$ and $10^{48} \mathrm{~s}^{-1}$ for a O7v and a B0v star (Vacca et al. 1996), we find from the observed fluxes of the $\mathrm{H} \beta$ emission line that the number of $\mathrm{O} 7 \mathrm{~V}$ and $\mathrm{B} 0 \mathrm{v}$ stars is in the range 2-7 and 23-70, respectively, depending on the location in the main body (see Table 4). Assuming a Salpeter IMF with a slope 2.35 , and upper and lower star mass limits of $120 M_{\odot}$ and $2 M_{\odot}$, we derive a total mass $10^{4}-10^{5} M_{\odot}$ for the young stellar population. Cerviño et al. (2000) have analyzed the stochastic nature of the IMF in young stellar clusters with solar metallicity. In particular, they find that in the range of equivalent widths $E W(\mathrm{H} \beta)=1-10 \AA$, the dispersion of age at fixed $E W(\mathrm{H} \beta)$ is not greater than $5 \%-10 \%$ if the total mass of the cluster lies in the range $10^{4}-10^{5} M_{\odot}$. Hence, we argue that stochastic effects in SBS $0940+544$ do not significantly change our age estimate from the hydrogen emission line equivalent widths. 
Table 4. Fluxes, equivalent widths of the $\mathrm{H} \alpha$ and $\mathrm{H} \beta$ emission lines and the extinction coefficient $C(\mathrm{H} \beta)$ in different regions of the SBS 0940+544 main body.

\begin{tabular}{lcccrrrrr}
\hline \hline Telescope & \# of region & Distance $^{a}$ & Aperture $^{b}$ & $F(\mathrm{H} \alpha)^{c}$ & $F(\mathrm{H} \beta)^{c}$ & $E W(\mathrm{H} \alpha)^{d}$ & $E W(\mathrm{H} \beta)^{d}$ & $C(\mathrm{H} \beta)$ \\
\hline Keck II $^{e}$ & 1 & 6.3 & $1.0 \times 3.0$ & $13.30 \pm 0.14$ & $3.91 \pm 0.12$ & $67.18 \pm 0.75$ & $12.37 \pm 0.24$ & $0.13 \pm 0.03$ \\
& 2 & 9.1 & $1.0 \times 2.6$ & $3.15 \pm 0.08$ & $0.72 \pm 0.08$ & $22.23 \pm 0.56$ & $3.26 \pm 0.24$ & $0.29 \pm 0.05$ \\
& 3 & 12.2 & $1.0 \times 3.6$ & $2.06 \pm 0.10$ & $0.43 \pm 0.08$ & $13.84 \pm 0.64$ & $2.18 \pm 0.31$ & $0.35 \pm 0.07$ \\
& 4 & 16.4 & $1.0 \times 4.8$ & $3.60 \pm 0.13$ & $1.05 \pm 0.12$ & $45.72 \pm 1.71$ & $9.15 \pm 0.73$ & $0.13 \pm 0.05$ \\
\hline MMT $^{e}$ & $1 \mathrm{a}$ & 6.3 & $1.5 \times 3.0$ & $14.91 \pm 0.39$ & $4.22 \pm 0.29$ & $88.14 \pm 2.32$ & $14.82 \pm 1.01$ & $0.15 \pm 0.04$ \\
& $2 \mathrm{a}$ & 9.0 & $1.5 \times 2.4$ & $3.53 \pm 0.17$ & $0.79 \pm 0.14$ & $28.76 \pm 1.41$ & $3.87 \pm 0.69$ & $0.30 \pm 0.06$ \\
& $3 \mathrm{a}$ & 12.6 & $1.5 \times 4.8$ & $3.05 \pm 0.31$ & $0.79 \pm 0.28$ & $16.10 \pm 1.62$ & $2.82 \pm 0.73$ & $0.21 \pm 0.08$ \\
& $4 \mathrm{a}$ & 16.8 & $1.5 \times 3.6$ & $2.96 \pm 0.24$ & $0.77 \pm 0.19$ & $41.86 \pm 3.41$ & $6.71 \pm 1.13$ & $0.21 \pm 0.09$ \\
\hline MMT $^{f}$ & 5 & 8.0 & $1.5 \times 10.8$ & $14.64 \pm 0.57$ & $3.65 \pm 0.39$ & $32.74 \pm 1.28$ & $6.40 \pm 0.45$ & $0.23 \pm 0.06$ \\
& 6 & 7.0 & $1.5 \times 7.2$ & $14.32 \pm 0.47$ & $4.00 \pm 0.50$ & $39.75 \pm 1.32$ & $5.97 \pm 0.48$ & $0.16 \pm 0.07$ \\
\hline \hline
\end{tabular}

${ }^{a}$ Distance in arcsec from the brightest $\mathrm{H}$ II region.

${ }^{b}$ Aperture $x \times y$ where $x$ is the slit width and $y$ the size along the slit in arcsec.

${ }^{c}$ In units $10^{-16} \mathrm{erg} \mathrm{s}^{-1} \mathrm{~cm}^{-2}$.

${ }^{d}$ In $\AA$.

${ }^{e}$ Slit orientation with position angle $\mathrm{PA}=-41^{\circ}$.

${ }^{f}$ Slit orientation with position angle $\mathrm{PA}=0^{\circ}$.

\subsubsection{Age from the hydrogen stellar absorption lines}

Another extinction-insensitive method for determining the age of stellar populations is based on equivalent widths of absorption features. It can be used for larger ages than the nebular emission line method because the longer-lived $\mathrm{B}$ and $\mathrm{A}$ stars show more prominent hydrogen absorption lines.

Gonzalez Delgádo \& Leitherer (1999) and Gonzalez Delgádo et al. (1999) calculated a grid of synthetic profiles of stellar hydrogen Balmer absorption lines for effective temperatures and gravities characteristic of starburst galaxies. They developed evolutionary stellar population synthesis models, synthesizing the profiles of the hydrogen Balmer absorption lines from $\mathrm{H} \beta$ to $\mathrm{H} 13$ for instantaneous bursts with ages ranging from $10^{6}$ to $10^{9} \mathrm{yr}$, in the case of a stellar initial mass function with a Salpeter slope and mass cutoffs $M_{\text {low }}=1 M_{\odot}$ and $M_{\text {up }}=80 M_{\odot}$. Their models predict a steady increase in the equivalent widths with age. However, at larger ages $\gtrsim 1$ Gyr the situation is the opposite and the equivalent widths of the absorption lines decrease with age (Bica \& Alloin 1986).

The hydrogen absorption lines due to the underlying stellar populations are seen clearly along the whole elongated main body of SBS $0940+544$. We show in Fig. 7 the $\mathrm{H} \gamma, \mathrm{H} \delta$ and other hydrogen absorption lines in two regions along the slit oriented with position angle $\mathrm{PA}=-41^{\circ}$. A weak K Ca II absorption line is also present. While the $\mathrm{H} \gamma$ nebular emission line is superimposed on the absorption line in region 1 , it is not seen in region 3 .

We measured the parameters of the $\mathrm{H} \gamma$ and $\mathrm{H} \delta$ absorption lines in 4 regions along the main body. A careful placement of the continuum is very important for deriving accurate $E W$ s. To define the continuum level, we

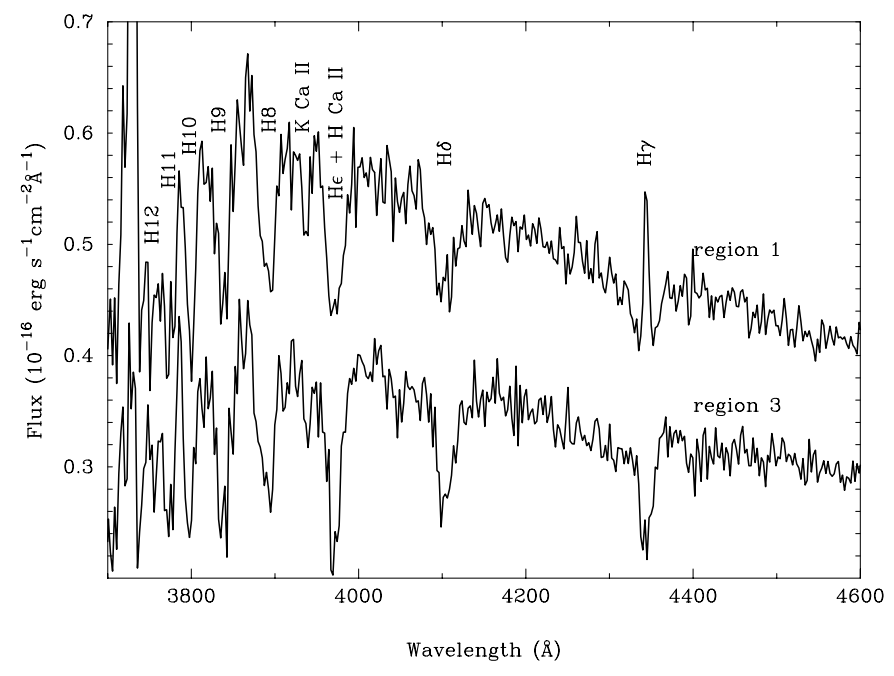

Fig. 7. Keck II spectra of regions 1 and 3 (respectively at 6.3 and $12^{\prime \prime} .2$ from the brightest $\mathrm{H}$ II region) showing $\mathrm{H} \gamma, \mathrm{H} \delta$ and other hydrogen and Ca II absorption lines.

select several points in the spectral regions free of nebular and stellar lines. The continuum is then fitted by cubic splines and the quality of the continuum fit is visually checked. Although the contamination of the absorption lines by nebular emission in regions 2, 3 and 4 is very small, not more than a few percent, we have corrected the absorption equivalent widths for it. For this purpose the expected fluxes of the $\mathrm{H} \gamma$ and $\mathrm{H} \delta$ emission lines have been calculated from the flux of the $\mathrm{H} \beta$ emission line. Then these fluxes have been subtracted from the negative fluxes of the absorption lines. Here we adopt theoretical recombination $\mathrm{H} \gamma / \mathrm{H} \beta$ and $\mathrm{H} \delta / \mathrm{H} \beta$ flux ratios at an electron temperature $T_{\mathrm{e}}=10^{4} \mathrm{~K}$ and an electron number density 
Table 5. Uncorrected and corrected equivalent widths of the $\mathrm{H} \gamma$ and $\mathrm{H} \delta$ absorption lines in the main body of SBS $0940+544$.

\begin{tabular}{lccccccc}
\hline \hline Telescope & \# of region & Distance $^{a}$ & Aperture $^{b}$ & $E W(\mathrm{H} \delta)^{c}$ & $E W(\mathrm{H} \gamma)^{c}$ & $E W(\mathrm{H} \delta)_{\text {cor }^{c}}$ & $E W(\mathrm{H} \gamma)_{\text {cor }^{c}}$ \\
\hline Keck II $^{d}$ & 1 & 6.3 & $1.0 \times 3.0$ & $4.51 \pm 0.22$ & $2.57 \pm 0.23$ & $6.41 \pm 0.49$ & $6.37 \pm 0.55$ \\
& 2 & 9.1 & $1.0 \times 2.6$ & $6.96 \pm 0.24$ & $5.47 \pm 0.48$ & $7.47 \pm 0.68$ & $6.50 \pm 0.77$ \\
& 3 & 12.2 & $1.0 \times 3.6$ & $6.65 \pm 0.29$ & $5.40 \pm 0.24$ & $6.95 \pm 0.47$ & $6.01 \pm 0.29$ \\
& 4 & 16.4 & $1.0 \times 4.8$ & $5.71 \pm 0.53$ & $5.40 \pm 0.41$ & $7.19 \pm 0.79$ & $6.40 \pm 0.41$ \\
\hline $\mathrm{MMT}^{d}$ & $1 \mathrm{a}$ & 6.3 & $1.5 \times 3.0$ & $3.95 \pm 0.50$ & $0.70 \pm 0.60$ & $5.99 \pm 0.78$ & $5.08 \pm 0.68$ \\
& $2 \mathrm{a}$ & 9.0 & $1.5 \times 2.4$ & $6.28 \pm 0.43$ & $4.50 \pm 0.47$ & $6.80 \pm 0.59$ & $5.61 \pm 0.55$ \\
& $3 \mathrm{a}$ & 12.6 & $1.5 \times 4.8$ & $7.63 \pm 0.63$ & $5.23 \pm 0.70$ & $8.00 \pm 0.69$ & $6.00 \pm 0.74$ \\
& $4 \mathrm{a}$ & 16.8 & $1.5 \times 3.6$ & $5.76 \pm 0.87$ & $5.01 \pm 0.52$ & $6.73 \pm 0.92$ & $5.81 \pm 0.68$ \\
\hline $\mathrm{MMT}^{e}$ & 5 & 8.0 & $1.5 \times 10.8$ & $5.85 \pm 0.53$ & $2.52 \pm 0.53$ & $6.86 \pm 0.65$ & $4.55 \pm 0.76$ \\
& 6 & 7.0 & $1.5 \times 7.2$ & $6.40 \pm 0.49$ & $2.71 \pm 0.52$ & $7.49 \pm 0.63$ & $4.92 \pm 0.88$ \\
\hline \hline
\end{tabular}

${ }^{a}$ Distance in arcsec from the brightest $\mathrm{H}$ II region.

${ }^{b}$ Aperture $x \times y$ where $x$ is the slit width and $y$ the size along the slit in arcsec.

${ }^{c}$ In $\AA$.

${ }^{d}$ Slit orientation with position angle $\mathrm{PA}=-41^{\circ}$.

${ }^{e}$ Slit orientation with position angle $\mathrm{PA}=0^{\circ}$.

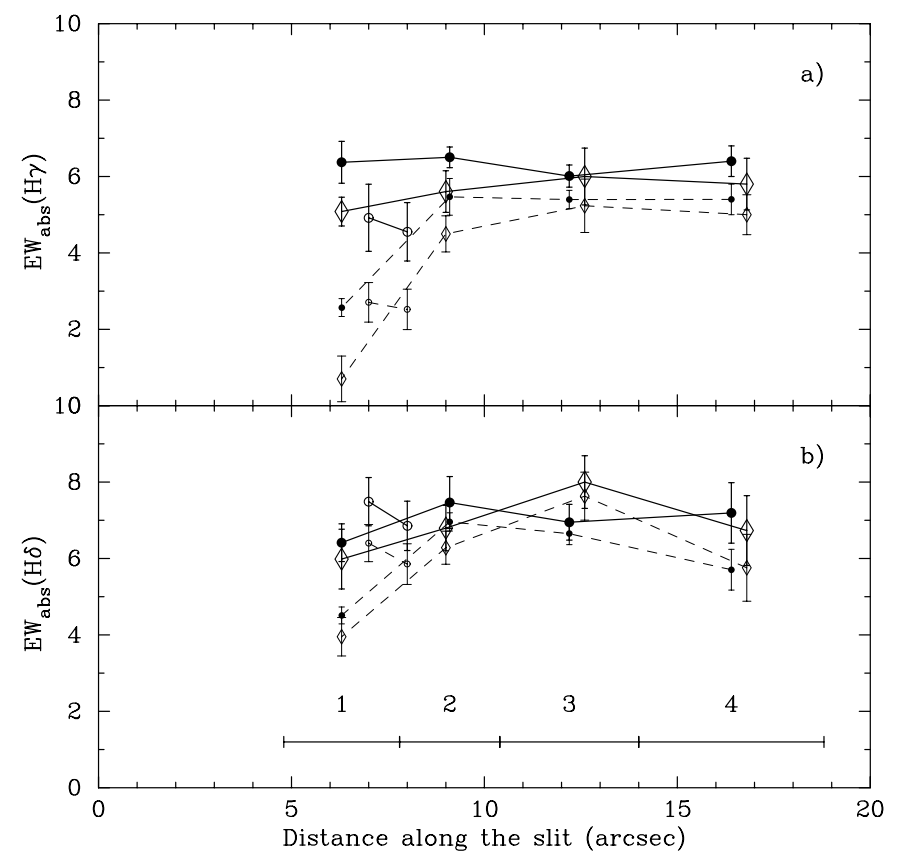

Fig. 8. The equivalent widths of $\mathrm{H} \gamma$ a) and $\mathrm{H} \delta$ b) absorption lines in 4 regions along the main body of SBS $0940+544$. Filled large circles, open large rhombs and solid lines show the corrected $E W_{\text {abs }}(\mathrm{H} \gamma)$ and $E W_{\text {abs }}(\mathrm{H} \delta)$ obtained respectively from the Keck II and MMT observations with position angle of the slit $\mathrm{PA}=-41^{\circ}$. Large open circles connected by solid lines show the data from the MMT spectrum with the slit oriented at $\mathrm{PA}=0^{\circ}$. Small symbols and dashed lines denote the equivalent widths uncorrected for emission.

$N_{\mathrm{e}}=10^{2} \mathrm{~cm}^{-3}$. We do not correct for extinction. Extinction correction would result in lower $E W_{\text {abs }}$ and smaller ages. Hence the ages derived in this section are upper limits.

We measured equivalent widths for only the $\mathrm{H} \gamma$ and $\mathrm{H} \delta$ absorption lines. Although higher-order hydrogen Balmer absorption lines are seen in the spectrum of the main body, they are not suitable for age determination because of (a) the relatively low signal-to-noise ratio of the spectra at short wavelengths and uncertainties in the placement of the continuum in the blue region due to many blended absorption features, and (b) the weak dependence of their equivalent widths on age (Gonzalez Delgádo et al. 1999). Additionally, the $\mathrm{H} \epsilon$ absorption line is blended with the H Ca II absorption line.

The measured and corrected equivalent widths of $\mathrm{H} \gamma$ and $\mathrm{H} \delta$ absorption lines in the Keck II and MMT spectra are listed in Table 5. The errors include uncertainties in the placement of the continuum. In Fig. 8 the measured (dashed lines) and corrected (solid lines) equivalent widths of the $\mathrm{H} \gamma$ and $\mathrm{H} \delta$ absorption lines are shown for the 4 regions along the main body of SBS $0940+544$. Note that the corrected equivalent widths of $\mathrm{H} \gamma$ and $\mathrm{H} \delta$ do not show statistically significant spatial variations.

Figure 9 shows the predicted behaviour of the equivalent widths of $\mathrm{H} \gamma$ (thick solid line) and $\mathrm{H} \delta$ (thick dashed line) absorption lines with age for an instantaneous burst at a metallicity $Z=1 / 20 Z_{\odot}$ from Gonzalez Delgádo et al. (1999) for ages $\leq 1$ Gyr and Bica \& Alloin (1986) for ages $>1$ Gyr. The upper and lower limits of the corrected $E W(\mathrm{H} \gamma)$ and $E W(\mathrm{H} \delta)$ in the 4 regions along the main body of the galaxy are shown respectively by thin solid and dotted horizontal lines. The shaded rectangular region gives age limits, ranging between 15 and $50 \mathrm{Myr}$. Though slightly larger, this age estimate is consistent with that obtained from the nebular emission line analysis, particularly since it constitutes an upper limit. Instantaneous burst models also predict low $E W(\mathrm{H} \gamma)$ and $E W(\mathrm{H} \delta)$ at age $\sim 10$ Gyr. However, this age is inconsistent with the one derived from the emission line equivalent widths. 


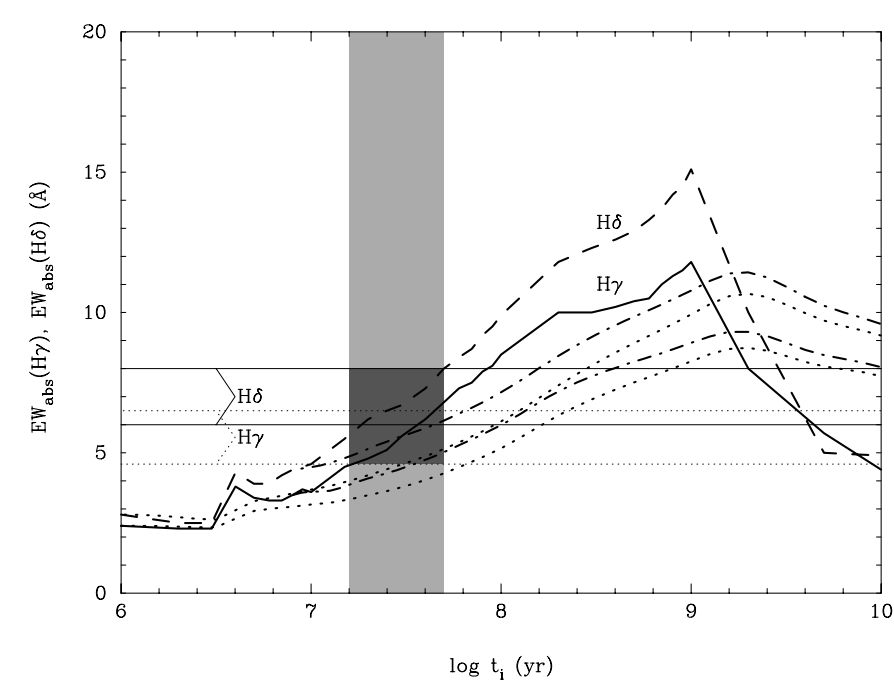

Fig. 9. Equivalent widths of $\mathrm{H} \gamma$ and $\mathrm{H} \delta$ absorption lines vs age for an instantaneous burst with metallicity $Z=1 / 20 Z_{\odot}$ from Gonzalez Delgádo et al. (1999) (for ages $\leq 1 \mathrm{Gyr}$ ) and Bica \& Alloin (1986) (for ages $>1$ Gyr), shown by thick solid and dashed lines respectively. The upper and lower limits of measured $E W(\mathrm{H} \gamma)$ and $E W(\mathrm{H} \delta)$ in 4 regions along the main body of the galaxy are shown by thin dotted and solid horizontal lines. Model predictions are also shown for the case of constant continuous star formation starting at an age defined by the abscissa $t_{\mathrm{i}}$ and stopping at $t_{\mathrm{f}}$, with $t_{\mathrm{f}}=0 \mathrm{Myr}$ (dotted lines), and $t_{\mathrm{f}}=8 \mathrm{Myr}$ (dash-dotted lines). The shaded rectangular region gives the age limits in the case of an instantaneous burst.

\subsubsection{Age from the spectral energy distribution}

Useful constraints on the ages of stellar populations can also be obtained from the spectral energy distribution (SED). As already pointed out, this method is subject to the effects of interstellar extinction. However, the combination of the spectral energy distribution method with the methods discussed in Sects. 5.1.1 and 5.1.2 can be used for a simultaneous derivation of the age and extinction.

To fit the observed SEDs, we use the galactic evolution code PEGASE.2 (Fioc \& Rocca-Volmerange 1997) to produce a grid of theoretical SEDs for an instantaneous burst of star formation and ages ranging between 0 and $10 \mathrm{Gyr}$, and a heavy element mass fraction of $Z=1 / 20 Z_{\odot}$. An initial mass function with a Salpeter slope $(\alpha=-2.35)$, and upper and lower mass limits of $120 M_{\odot}$ and $0.1 M_{\odot}$ are adopted. Because the equivalent widths of hydrogen emission lines in all 4 regions along the main body are small, the contribution of the ionized gas emission is not significant. For this reason we do not include gaseous emission in the SED calculations. Hence, photometric and spectroscopic data give us direct information on stellar populations if the interstellar extinction is known.

The extinction in each region can be estimated from the observed decrement of the Balmer emission lines. Many strong hydrogen emission lines are present in the brightest H II region a (Fig. 5) which allow a precise determination of the extinction. From the Keck II and

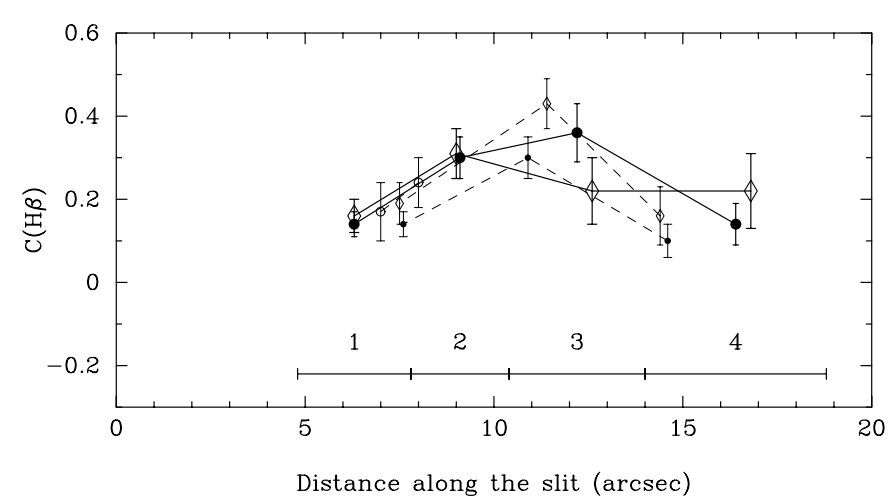

Fig. 10. Extinction coefficient $C(\mathrm{H} \beta)$, derived from the flux ratios of the emission lines $\mathrm{H} \alpha$ and $\mathrm{H} \beta$ in 4 regions along the main body. Filled large circles, open large rhombs and solid lines show data respectively from the Keck II and MMT spectra for the slit oriented at position angle $\mathrm{PA}=-41^{\circ}$. Large open circles show data from the MMT spectra with the slit oriented at position angle $\mathrm{PA}=0^{\circ}$. Small symbols and dashed lines show the same data, but for 3 regions in larger apertures located at $7^{\prime \prime} 6,10^{\prime \prime} 9$ and $14^{\prime \prime} .6$ from the brightest $\mathrm{H}$ II region with respective apertures $1^{\prime \prime} 0 \times 5$.' 6,1 .' $0 \times 6$.' 2 and $1^{\prime \prime} .0 \times 8^{\prime \prime} .4$ in the Keck II spectra and 1." $5 \times 5$ ". $6,1^{\prime \prime} .5 \times 6$ ". 2 and $1^{\prime \prime} .5 \times 88^{\prime \prime} .4$ in the MMT spectra.

MMT spectra of this region we derive very low values of $C(\mathrm{H} \beta), 0.08 \pm 0.02$ and $0.00 \pm 0.01$ respectively. The $\mathrm{H} \alpha$ and $\mathrm{H} \beta$ emission lines are also present in the main body, though weaker. We use the fluxes of these lines to derive $C(\mathrm{H} \beta)$ in different regions along the slit. The results are shown in Table 4 and in Fig. 10. We note that, in general, the extinction in the main body, derived from the observed Balmer decrement, is significantly larger than that in the brightest $\mathrm{H}$ II region, with a maximum $C(\mathrm{H} \beta) \sim 0.3$ in region 3 at a distance of $\sim 12^{\prime \prime}$ (or $\sim 1.5 \mathrm{kpc}$ ) from the brightest $\mathrm{H}$ II region.

In principle, the extinction can also be derived from the SEDs of regions 1-4, adopting the age of the stellar population derived from the equivalent widths of the hydrogen emission and absorption lines. First we assume $C(\mathrm{H} \beta)=0$. Then the observed SEDs are redder than the theoretical SED of a stellar population with age $t=$ $20 \mathrm{Myr}$ (lower spectra in Figs. 11a-d). One of the reasons for the difference between the observed and theoretical SEDs is that interstellar extinction in the main body is not negligible and it modifies the observed spectral energy distribution. To derive $C(\mathrm{H} \beta)$ we adopt an age of the stellar population in each of the regions 1-4 equal to $20 \mathrm{Myr}$, as a representative value derived from the hydrogen emission and absorption line equivalent widths. We then compute the extinction coefficient $C(\mathrm{H} \beta)$ to achieve the best agreement between the observed SED after correction for interstellar extinction and the theoretical SED. The observed extinction-corrected SEDs are superimposed on the synthetic $20 \mathrm{Myr}$ stellar population SED for regions 1 to 4 in Figs. 11a-d (upper spectra). They are labeled by the derived values of $C(\mathrm{H} \beta)$. These values are consistent with those derived from the $\mathrm{H} \alpha / \mathrm{H} \beta$ flux ratios (Table 4, 


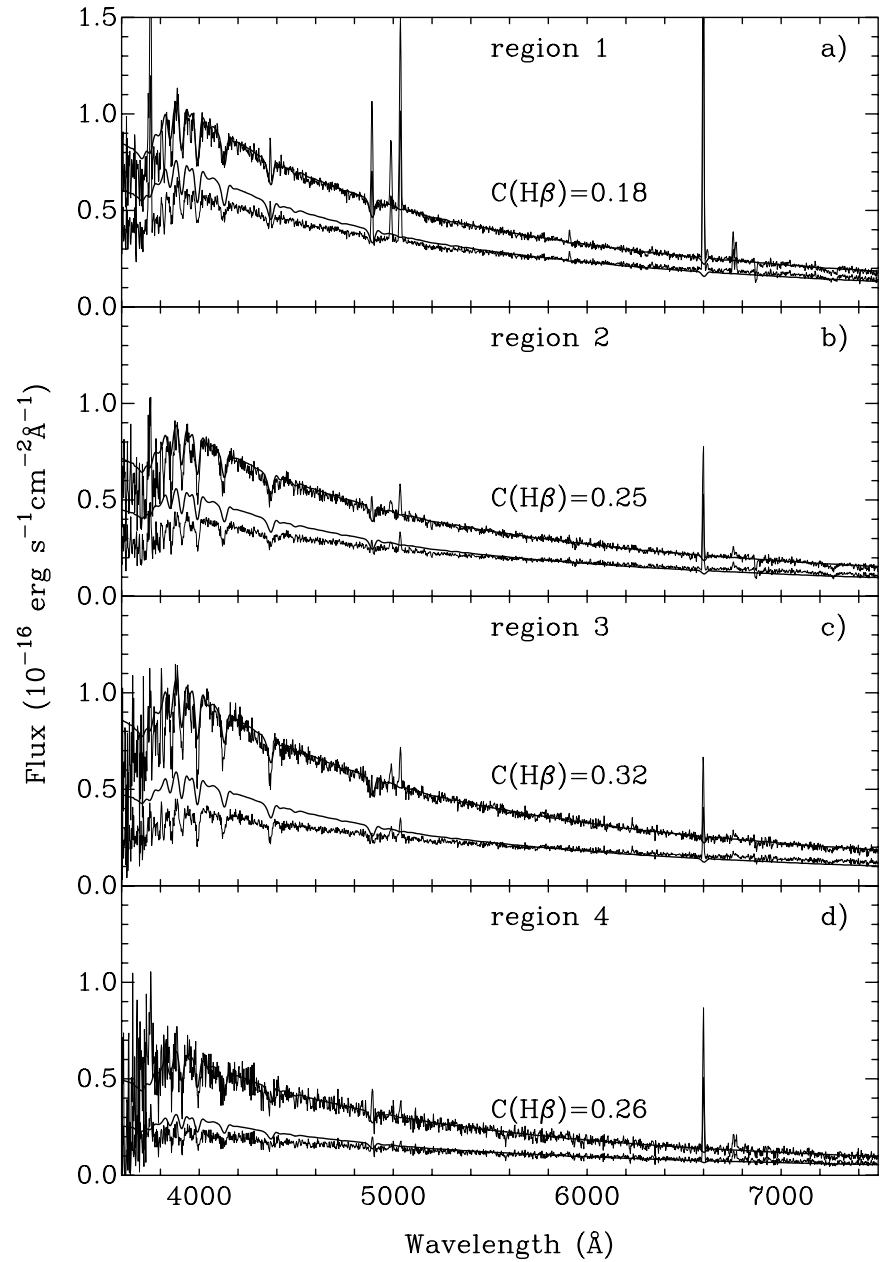

Fig. 11. Keck II telescope spectra of 4 regions in the main body of SBS $0940+544$ on which synthetic continuum spectral energy distributions are superposed. Lower spectra in a)-d) are synthetic SEDs of a 20 Myr instantaneous burst stellar population superposed on the observed spectra uncorrected for extinction $(C(\mathrm{H} \beta)=0)$. Upper spectra in $\mathbf{a})-\mathbf{d})$ are synthetic SEDs of a 20 Myr instantaneous burst stellar population superposed on the spectra corrected for extinction. Each upper spectrum is labeled by the extinction coefficient $C(\mathrm{H} \beta)$ which gives the best fit of the theoretical SED to the extinction-corrected observed SED.

Fig. 10). However, despite this consistency, it is seen from Fig. 11, that the observed SEDs (upper spectra) at $\lambda \lesssim$ $3900 \AA$ are not well fitted by the theoretical SEDs. Hence, we conclude that the instantaneous burst models do not adequately reproduce the observed properties of the SBS 0940+544 main body.

\subsection{The case of continuous star formation}

Our estimates for the stellar population age in SBS 0940+544 made in Sect. 5.1 are based on the assumption of an instantaneous burst of star formation. Now we consider how the age of the stellar population is changed if star formation is continuous. For this we adopt a constant star formation rate in the interval between the time $t_{\mathrm{i}}$ when star formation starts and $t_{\mathrm{f}}$ when it stops. Time is zero now and increases to the past. It is customary to consider models with a constant star formation rate, with star formation continuing until now, i.e. with $t_{\mathrm{f}}=$ 0 . However, we will also consider models with $t_{\mathrm{f}}>0$ because of the following reason. The number of the ionizing photons in regions $1-4$ derived from the $\mathrm{H} \alpha$ luminosity is in the range $N(\mathrm{Lyc})=(2-7) \times 10^{49} \mathrm{~s}^{-1}$, several times lower than $N$ (Lyc) emitted by a single star with mass $\sim 100 M_{\odot}$ (Vacca et al. 1996). Hence, an IMF with a high $M_{\text {up }}$ does not strictly apply. There are however several circumstances which may explain the lack of ionizing photons: (a) stochastic effects on the IMF; (b) the major part (more than 50\%) of the ionizing photons escapes the $\mathrm{H}$ II region or is absorbed by dust; (c) the IMF upper mass limit is truncated at $\sim 30 M_{\odot}$; (d) star formation in regions 1-4 stopped several Myr ago, i.e. $t_{\mathrm{f}}>0$. We have considered all of these possibilities (except for (a)) in fitting the data and found that the result is not changed significantly. Therefore, in the subsequent discussion we use models with $M_{\text {up }}=120 M_{\odot}$ and $t_{\mathrm{f}} \geq 0$.

We use the model equivalent widths of hydrogen emission and absorption lines and SEDs for instantaneous bursts (Fioc \& Rocca-Volmerange 1997; Gonzalez Delgádo et al. 1999; Bica \& Alloin 1986) to calculate the temporal evolution of the equivalent widths of hydrogen emission and absorption lines in the case of continuous constant star formation. The results are given in Figs. 6 and 9. The temporal dependences of the equivalent widths of the $\mathrm{H} \beta$ and $\mathrm{H} \alpha$ emission lines (Fig. 6), and the $\mathrm{H} \delta$ and $\mathrm{H} \gamma$ absorption lines (Fig. 9) are shown for continuous star formation starting at time $t_{\mathrm{i}}$, as defined by the abscissa value, and stopping at $t_{\mathrm{f}}=0 \mathrm{Myr}$ (dotted line) and $t_{\mathrm{f}}=8 \mathrm{Myr}$ (dash-dotted line). The equivalent widths of the $\mathrm{H} \beta$ and $\mathrm{H} \alpha$ emission lines and of the $\mathrm{H} \delta$ and $\mathrm{H} \gamma$ absorption lines in the spectrum of the stellar population formed between $t_{\mathrm{i}}$ and $t_{\mathrm{f}}$ are represented in Figs. 6 and 9 by the values of $E W$ at time $t_{\mathrm{i}}$. At a fixed $E W$, it is seen that the younger the youngest stars, the larger the time interval $t_{\mathrm{i}}-t_{\mathrm{f}}$, i.e. the older the oldest stars.

\subsubsection{Continuous star formation with young stellar population}

Can the observed SEDs of regions 1-4 be fitted with only a young stellar population continuously formed over the last 100 Myr? To fit the observed SEDs and derive the extinction in regions 1-4 of the main body in the case of continuous star formation, we consider star formation occurring between $t_{\mathrm{f}}=8 \mathrm{Myr}$ and $t_{\mathrm{i}}=100 \mathrm{Myr}$. This model predicts $E W(\mathrm{H} \delta)=7.2 \AA, E W(\mathrm{H} \gamma)=6.0 \AA$, and $E W(\mathrm{H} \beta)=3.5 \AA, E W(\mathrm{H} \alpha)=21.2 \AA$, close to the values observed in regions 2 and 3 (Tables 4 and 5 ). However, the observed emission line equivalent widths in regions 1 and 4 are larger than the predicted values. For these two regions, a more appropriate model is that with $t_{\mathrm{i}}<100 \mathrm{Myr}$ and/or $t_{\mathrm{f}}<8 \mathrm{Myr}$. We show in Fig. 12 the results of our 


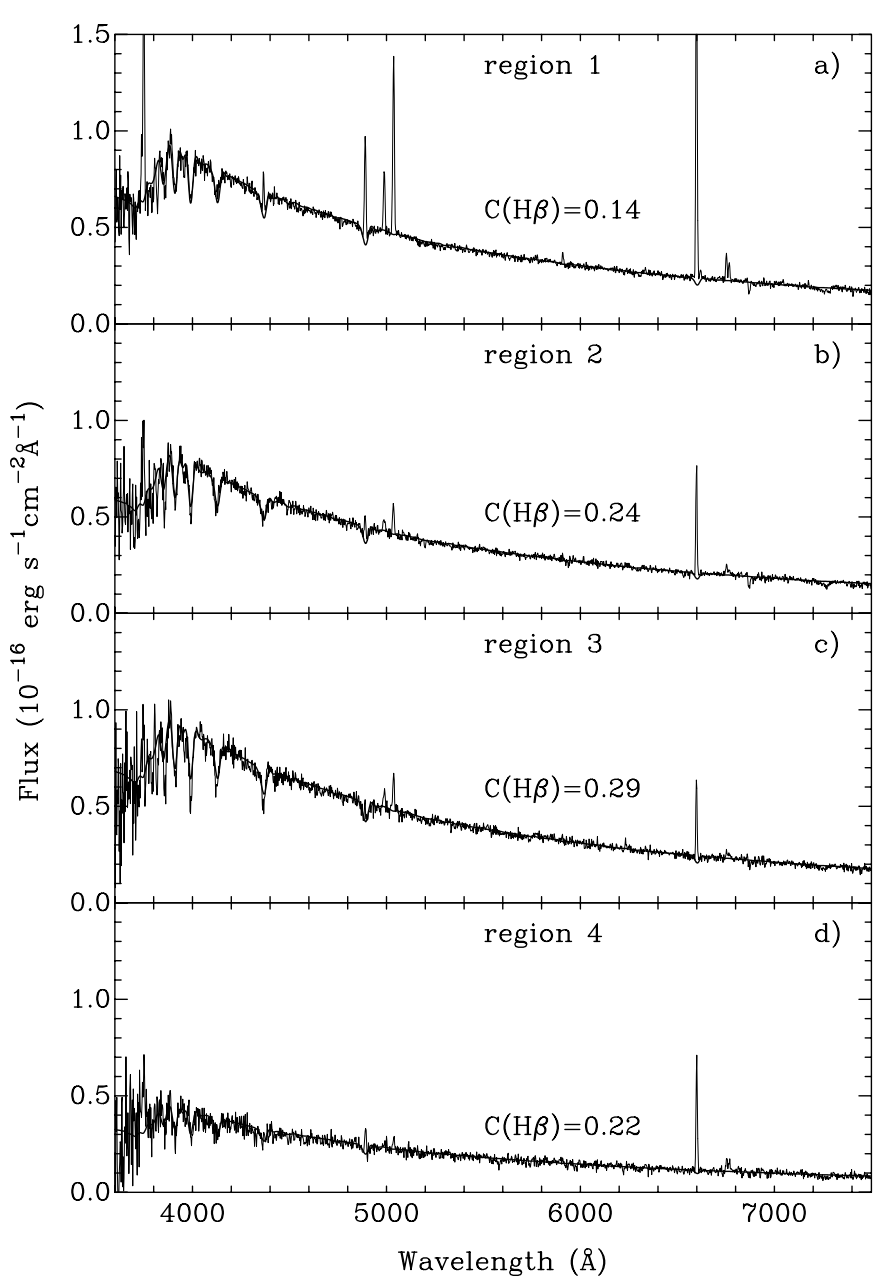

Fig. 12. Keck II telescope spectra of 4 regions along the main body of SBS $0940+544$ on which synthetic continuum spectral energy distributions are superposed. Spectra in a)-d) are synthetic SEDs of a stellar population continuously formed with constant star formation rate between 8 and $100 \mathrm{Myr}$ ago. These SEDs are superposed on the spectra corrected for extinction. Each spectrum is labeled by the extinction coefficient $C(\mathrm{H} \beta)$ which gives the best fit of the theoretical SED to the extinctioncorrected observed SED.

fitting. As in the case of an instantaneous burst, we adjust the extinction coefficient $C(\mathrm{H} \beta)$ to achieve the best agreement between the observed SED after correction for interstellar extinction and the theoretical SED. The observed extinction-corrected SEDs are superimposed on the synthetic stellar population SED for regions 1 to 4 in Figs. $12 \mathrm{a}-\mathrm{d}$. They are labeled by the derived values of $C(\mathrm{H} \beta)$. These values are in agreement with those derived from the $\mathrm{H} \alpha / \mathrm{H} \beta$ flux ratio. Note that the synthetic SEDs with continuous star formation reproduce better the observed spectra in the spectral range $\lambda<3900 \AA$ than those calculated on the assumption of an instantaneous burst. This makes continuous star formation in the main body of SBS $0940+544$, occuring during the last $100 \mathrm{Myr}$, a possible interpretation.

\subsubsection{Continuous star formation including old stellar population}

We next consider different continuous star formation scenarios in which an old stellar population is present in the main body of SBS $0940+544$. For this, we again adopt $C(\mathrm{H} \beta)=0$ and consider models with constant and varying $S F R$ s with ages ranging between 0 and 10 Gyr. Specifically, for a varying $S F R$, we consider two periods of continuous star formation with constant but different $S F R$ s, occurring in the age interval $\leq 100$ Myr and $>100 \mathrm{Myr}$. To quantify the recent-to-past star formation rate ratio, we introduce the parameter $b=S F R(t \leq$ $100 \mathrm{Myr}) / S F R(t>100 \mathrm{Myr})$. First we consider models with constant star formation during the whole 0-10 Gyr range, i.e. models with $b=1$. In Figs. $13 \mathrm{a}-\mathrm{d}$ we show such SEDs superimposed on the observed spectra of regions 14. It is evident that these models do not reproduce the observed SEDs. However, by increasing the parameter $b$ we can fit the observed SEDs. These theoretical SEDs are labeled by $b>1$ in Figs. 13a-d. The predicted equivalent widths of the emission and absorption hydrogen lines are also in agreement with the observed ones. If non-negligible extinction is present in regions 1-4 then to fit the observed SEDs, the parameter $b$ should be further increased. In particular, if the extinction $C(\mathrm{H} \beta)=0.28$ derived for region 2 from the $\mathrm{H} \alpha / \mathrm{H} \beta$ flux ratio is adopted then the parameter $b$ should be as high as $\sim 100$ to fit observations.

In summary, analysis of the spectroscopic data for the main body of SBS $0940+544$ shows that the stellar population can be equally well reproduced by a model of continuous star formation during the last $100 \mathrm{Myr}$, or by a model in which stars are continuously formed in the period 0-10 Gyr. In the former case, the extinction, as derived from the $\mathrm{H} \alpha / \mathrm{H} \beta$ flux ratio, should be taken into account. In the latter case, a fit to the observed SED is only possible when $b>5$, i.e. the star formation rate in the main body of SBS $0940+544$ has significantly increased over the last 100 Myr.

\subsubsection{Age of the low-surface-brightness host}

An upper limit to the age of the stellar population in SBS $0940+544$ can in principle be derived from photometric and spectroscopic measurements of the faint $\left(\mu_{V} \approx 24-\right.$ $24.5 \mathrm{mag} \operatorname{arcsec}^{-2}$ ) region $d$ (cf. Fig. 1a).

As discussed in Sect. 3.1 the $V-I$ and $V-R$ colours in this region are only slightly redder than the average respective values of $0.58 \mathrm{mag}$ and $0.33 \mathrm{mag}$ derived for the outskirts of SBS $0940+544$ from Fig. 4, thus it may be considered representative for the stellar LSB component.

The spectrum of region $d$ extracted from the MMT \#2 spectrum (position angle $\mathrm{PA}=0^{\circ}$ ) (cf. Fig. 1b) within a $4^{\prime \prime} .8 \times 1{ }^{\prime \prime} 5$ aperture is shown in Fig. 14 and labeled "1". Because of its very low intensity, the spectrum is smoothed by a 5-point box-car. No appreciable emission or absorption lines are seen in the spectrum, probably because of the low signal-to-noise ratio. Therefore, we cannot 


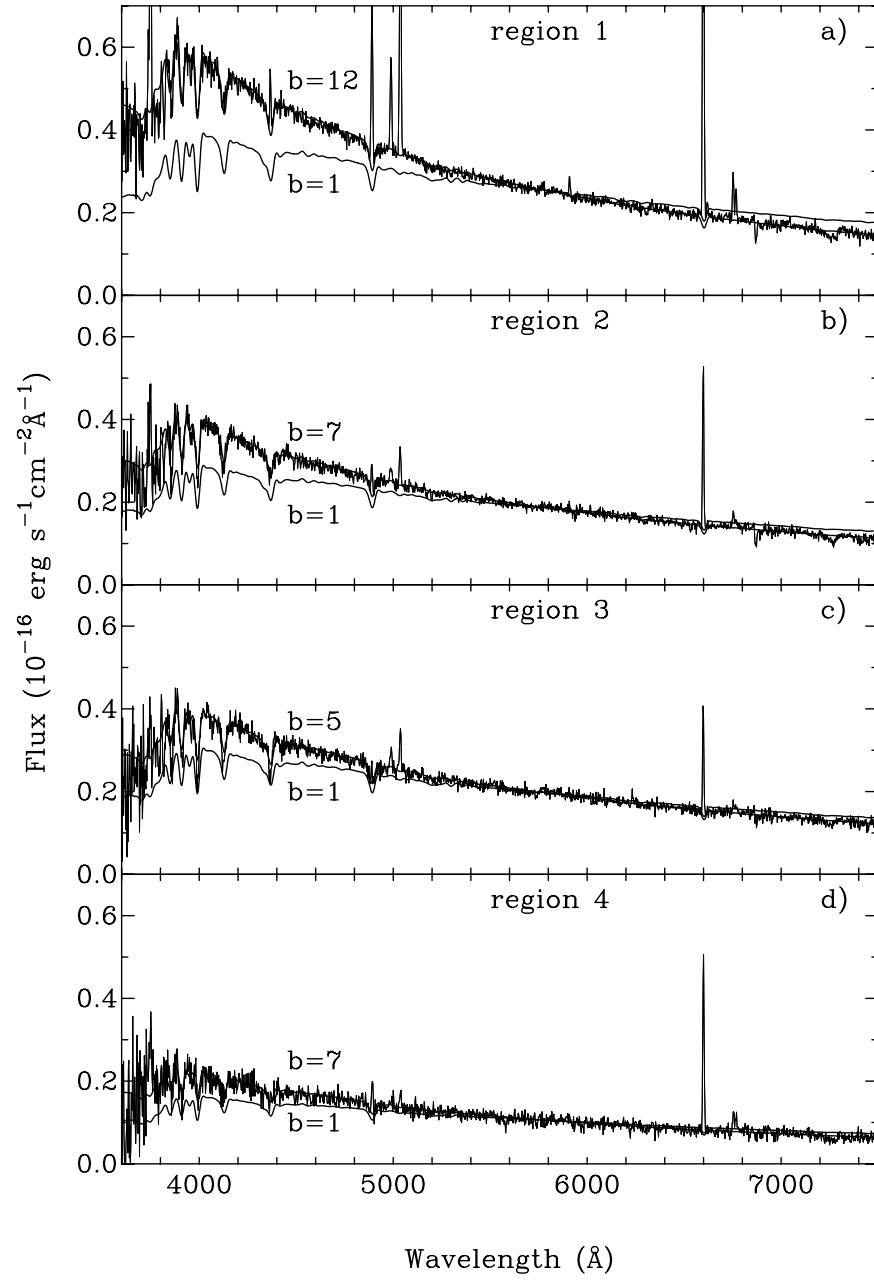

Fig. 13. Keck II telescope spectra of 4 regions in the main body of SBS $0940+544$ on which synthetic continuum spectral energy distributions are superposed. Synthetic SEDs shown in panels a) $-\mathbf{d})$ and labeled by $b \equiv S F R(t \leq$ $100 \mathrm{Myr}) / S F R(t>100 \mathrm{Myr})=1$ correspond to stellar populations formed continuously with a constant star formation rate between 0 and 10 Gyr. Synthetic spectra labeled by $b>1$ correspond to stellar populations formed continuously between 0 and 10 Gyr with a star formation rate enhanced by a factor of $b$ during the last 100 Myr. These SEDs are superposed on the spectra uncorrected for extinction.

estimate an age of the stellar populations in region $d$ by extinction-independent methods, or decide on whether the red colours of this region are caused by enhanced extinction or by an intrinsically red stellar population. Spectrum "1", when not corrected for extinction, is fitted by a theoretical SED of a stellar population continuously formed with constant star formation rate between 100 Myr and 10 Gyr ago.

However, in the presence of extinction the range of ages can be increased or the upper age limit can be significantly reduced. If a value of $C(\mathrm{H} \beta)=0.1$ is assumed for region $d$ then the corrected spectrum (labeled " 2 " in Fig. 14) is fitted by a theoretical SED of a stellar population continuously formed with constant star formation rate between 20 Myr and 10 Gyr ago. However, it is unlikely to

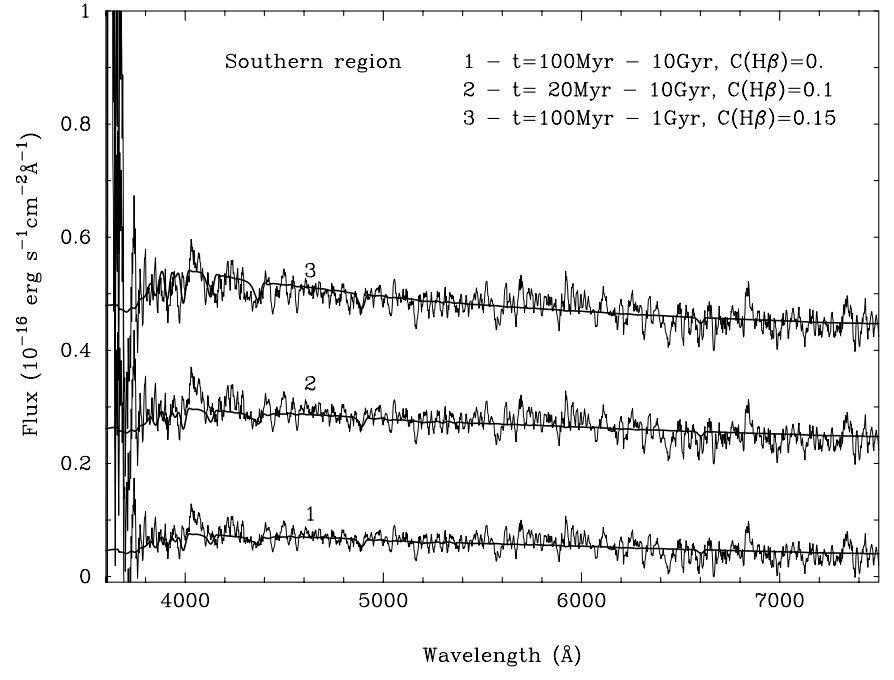

Fig. 14. MMT spectrum of region $d$ in the LSB host of SBS $0940+544$, smoothed by a 5 -point box-car and labeled as " 1 ". The spectrum is extracted from the two-dimensional spectrum obtained with the slit oriented at position angle $\mathrm{PA}=0^{\circ}$ in an aperture $4 . \prime .8 \times 1$." 5 . A theoretical SED of a stellar population continuously formed between $100 \mathrm{Myr}$ and $10 \mathrm{Gyr}$ ago is superimposed on " 1 ". The spectra labeled as "2" and " 3 " are the same spectra corrected for extinction respectively with $C(\mathrm{H} \beta)=0.1$ and 0.15 and superposed on a theoretical SEDs of a stellar population continuously formed between $100 \mathrm{Myr}$ and 1 Gyr ago and $20 \mathrm{Myr}$ and 1 Gyr ago. The latter two spectra and SEDs are offset up by $2 \times 10^{-17} \mathrm{erg} \mathrm{s}^{-1} \mathrm{~cm}^{-2} \AA^{-1}$ and $4 \times 10^{-17} \mathrm{erg} \mathrm{s}^{-1} \mathrm{~cm}^{-2} \AA^{-1}$.

reduce the lower age limit to values less than 10-15 Myr, otherwise emission lines must be present in the spectrum of region $d$. If a value of $C(\mathrm{H} \beta)=0.15$ is assumed then the corrected spectrum (labeled " 3 " in Fig. 14) is fitted by the theoretical SED of a stellar population continuously formed with a constant star formation rate between 100 Myr and 1 Gyr ago. The poor signal-to-noise ratio of the spectrum of region $d$ precludes reliable estimates of the age for this region.

\subsubsection{Age from the surface brightness and colour distributions}

As emphasized in the previous discussion, photometric data alone do not allow to draw definite conclusions on the age of the stellar populations. However, they can provide a consistency check of the stellar population ages derived from the spectra. Are the ages of the stellar populations derived above compatible with the broad-band colours?

We derived $V$ and $I$ surface brightness and colour distributions for the regions covered by the spectroscopic observations at both slit orientations and compared them with predictions from our population synthesis modeling. The results of this comparison are shown in Fig. 15. The predicted colours obtained from convolving the theoretical SEDs with the appropriate filter bandpasses are shown by different symbols. The transmission curves for 

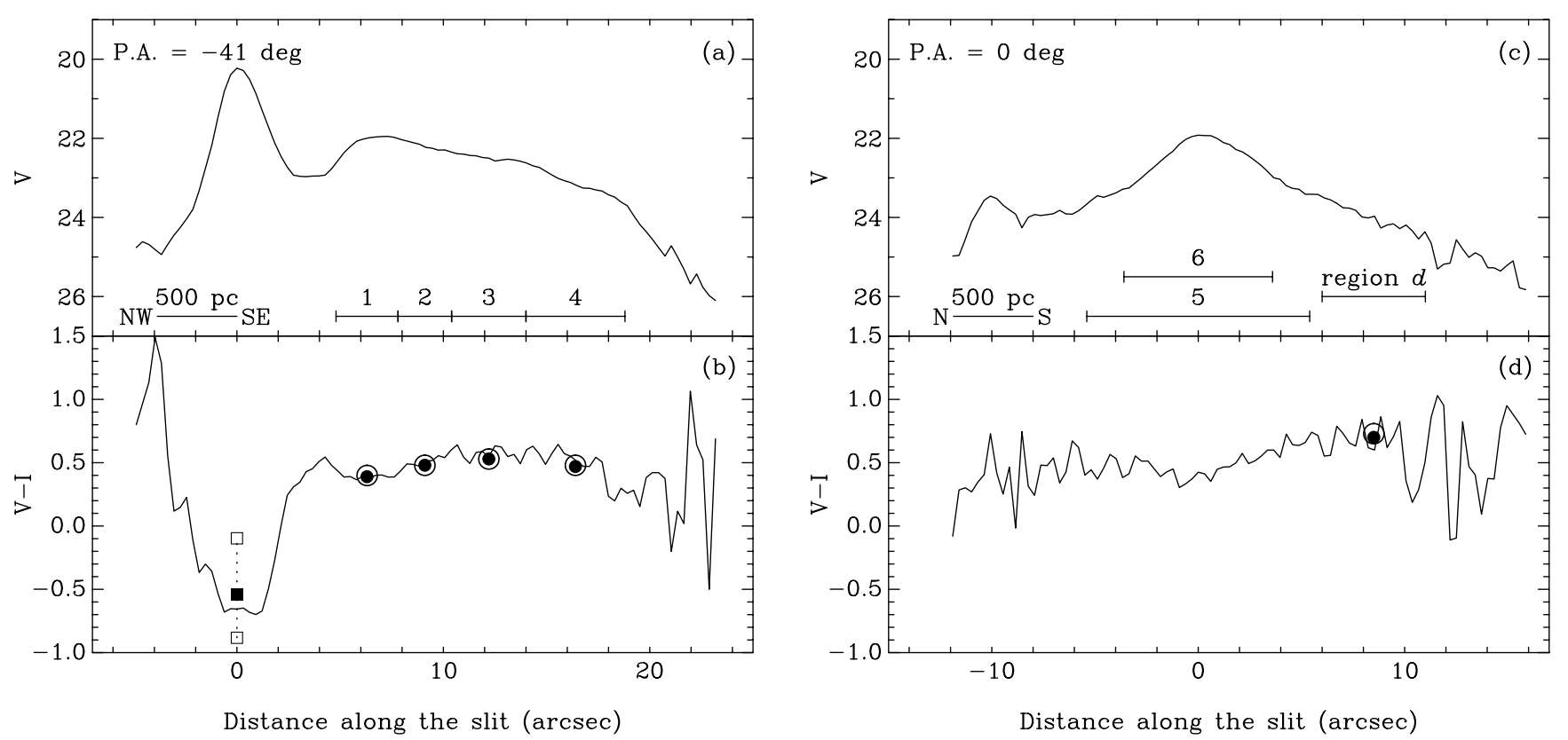

Fig. 15. a) The $V$ surface brightness distribution along the slit oriented at position angle $\mathrm{PA}=-41^{\circ}$. The origin is at the location of the brightest $\mathrm{H}$ II region. The regions used for spectroscopic analysis are labeled in a) "1" to " 4 ". b) The $V-I$ colour distribution along the slit with position angle $\mathrm{PA}=-41^{\circ}$. Filled circles are the predicted colours of a stellar population continuously formed with a constant star formation rate between $8 \mathrm{Myr}$ and $100 \mathrm{Myr}$ ago and reddened with an extinction coefficient as derived for each region in Fig. 12, open circles are the predicted colours of the stellar populations continuously formed between 0 and 10 Gyr with the enhancement parameters $b$ as derived for each region in Fig. 13. The filled square indicates the colour of a 4 Myr stellar population to which has been added the observed ionized gas emission in the brightest $\mathrm{H}$ II region. The upper open square shows the colour of a pure $4 \mathrm{Myr}$ stellar population, while the lower open square shows the colour of pure ionized gas emission. c) The $V$ surface brightness distribution along the slit oriented at position angle PA $=$ $0^{\circ}$. The origin is at the location of the surface brightness maximum in the main body coincident with region $c$ in Fig. 1 . The regions studied spectroscopically are labeled as " 5 " and "6". The location of the reddest part of SBS $0940+544$ is marked as "region $d$ ". d) The $V-I$ colour distribution along the slit with position angle PA $=0^{\circ}$. The filled circle shows the predicted colour of a stellar population continuously formed with constant star formation rate and reddened with an extinction coefficient $C(\mathrm{H} \beta)=0.15$ as derived for the reddest region in Fig. 14 (spectrum 3) while the open circle shows the predicted colour of a stellar population continuously formed with constant star formation rate and reddened with an extinction coefficient $C(\mathrm{H} \beta)=$ 0.1 as derived for the reddest region in Fig. 14 (spectrum 2).

the Johnson $V$ and Cousins $I$ bands are taken from Bessel (1990). The zero points are from Bessel et al. (1998).

Since the contribution of the ionized gas emission to the total brightness of the brightest H II region $(a)$ of SBS $0940+544$ is high, the theoretical SED for this region has been constructed in the following way. We have used the 4 Myr stellar population SED for a heavy element mass fraction $Z=Z_{\odot} / 20$. The observed gaseous SED is then added to the calculated stellar SED, its contribution being determined by the ratio of the observed equivalent width of the $\mathrm{H} \beta$ emission line to the one expected for pure gaseous emission. To calculate the gaseous continuum $\mathrm{SED}$, the observed $\mathrm{H} \beta$ flux and the electron temperature have been derived from the optical spectrum (Tables 2 and $3)$. The contribution of the free-bound, free-free and twophoton continuum emission has been taken into account for the spectral range from 0 to $5 \mu \mathrm{m}$ (Aller 1984; Ferland 1980). Emission lines are superposed on the gaseous continuum SED with intensities derived from spectra in the spectral range $\lambda 3700-7500 \AA$. Outside this range, the intensities of emission lines (mainly hydrogen lines) have been calculated from the extinction-corrected flux of $\mathrm{H} \beta$.

As evident from Fig. 15b the observed colour of the brightest $\mathrm{H}$ II region is very blue $V-I \sim-0.6 \mathrm{mag}$ and cannot be reproduced by a stellar population of any age. In particular, the $V-I$ colour equal to $-0.1 \mathrm{mag}$ of a $4 \mathrm{Myr}$ old stellar population (upper open square) is significantly redder than that observed. However, the synthetic colour of a $4 \mathrm{Myr}$ stellar population together with the ionized gas (filled square) is very similar to the observed colour. We also show by the lower open square the colour of pure ionized gas emission. It is very blue $(V-I \sim-0.9 \mathrm{mag})$ if the observed intensities of the emission lines (Table 2) and the redshift of SBS $0940+544$ are taken into account.

We already pointed out that the contribution of the gaseous emission is small in the main body. Therefore, we do not take it into account and consider the colours of a stellar population continuously formed with a constant star formation rate between 8 Myr and 100 Myr ago and reddened by interstellar extinction. In Fig. 15b we show by filled circles the colours of four regions with extinction 
coefficients derived from the best of the theoretical fits to the observed extinction-corrected spectra (spectra in Figs. 12a-d). Open circles show the predicted colours of a stellar population continuously formed between 0 and 10 Gyr ago, assuming enhanced star formation during last 100 Myr, as defined by parameter $b$ in Figs. 13a-d (upper spectra). The agreement between the $V-I$ colours obtained from the photometric data and those derived from the spectral energy distributions in both scenarios is very good.

We also compare the colour of the southern region $d$ (Fig. 15d) with predictions. Because of the noisy spectrum and large photometric errors for this region a broad range of ages can be consistent with the observed colour. In particular, the colour of the stellar population continuously formed with a constant star formation rate between $100 \mathrm{Myr}$ and 1 Gyr ago and reddened with extinction $C(\mathrm{H} \beta)=0.15$ is shown in Fig. 15d by the filled circle, while the colour of the stellar population continuously formed with a constant star formation rate between $20 \mathrm{Myr}$ and 10 Gyr ago and reddened with extinction $C(\mathrm{H} \beta)=0.1$ is shown by the open circle. They are in good agreement with the observed colour for this region.

Finally, an upper age limit for stellar populations in SBS $0940+544$ can be estimated from the $V-R$ and $V-I$ colours of the outermost regions without spectroscopic observations. The mean colours in the outskirts of the LSB component of $V-R=0.33 \pm 0.04$ and $V-I=0.58 \pm 0.03$ (Fig. 4) are compatible with those of a stellar population forming continuously for the last several Gyr and assuming that star formation continues until now. However, the spatial distributions of the old and young stellar populations inferred from HST colour-magnitude diagrams of some galaxies, are different (e.g. Schulte-Ladbeck et al. 1998). The oldest stars are seen at largest distances, where younger stars are not detected. It is likely that stars in these extended haloes were formed during the galaxy formation era and no new stars have formed since then, or stars have diffused to haloes from the inner regions where they were born. If this is the case for the outermost regions of SBS $0940+544$, then to explain their observed $V-R$ and $V-I$ colours, the upper age limit should be significantly reduced, because no young stellar population is present there. In that case, the observed colours would be compatible with an upper age limit of $\leq 1$ Gyr for the stellar LSB component.

\section{Conclusions}

We present in this paper a detailed photometric and spectroscopic study of the very metal-deficient blue compact dwarf galaxy SBS 0940+544 $\left(Z \sim Z_{\odot} / 27\right)$, a good young galaxy candidate. Photometric $V$ and $I$ data have been obtained with the $2.1 \mathrm{~m}$ Kitt Peak telescope, and $R$ imaging has been done with the $1.23 \mathrm{~m}$ Calar Alto telescope. Very high signal-to-noise spectra in the optical range have been obtained with the $10 \mathrm{~m}$ Keck II telescope and the
$4.5 \mathrm{~m} \mathrm{MMT}$ at two slit orientations. We have reached the following conclusions:

1. SBS $0940+544$ is a nearby BCD galaxy with cometarylike structure, i.e. a bright off-center H II region and an elongated main body. The $V, R$ and $I$ surface brightness profiles of the galaxy's main body are very similar, with an exponential scale length $\alpha \sim 320$ pc. The $V-I$ colour of the brightest $\mathrm{H}$ II region is very blue $\sim-0.6 \mathrm{mag}$, due to the combined effect of the young stellar population and the ionized gas emission. The colours of the main body are much redder, without significant gradients;

2. The derived oxygen abundance $12+\log (\mathrm{O} / \mathrm{H})=$ 7.46-7.50 is consistent within the errors with previous abundance determinations. The $\alpha$-element $\mathrm{Ne} / \mathrm{O}$, $\mathrm{S} / \mathrm{O}, \mathrm{Ar} / \mathrm{O}$ abundance ratios are in good agreement with the mean ratios derived from previous studies of BCDs (Izotov \& Thuan 1999). The nitrogen-to-oxygen abundance ratio $\log \mathrm{N} / \mathrm{O}=-1.60--1.65$ lies in the narrow range obtained by Thuan et al. (1995) and Izotov \& Thuan (1999) for the most metal-deficient BCDs. These abundances suggest that SBS 0940+544 is a good local young galaxy candidate;

3. The ${ }^{4} \mathrm{He}$ mass fractions $Y=0.247 \pm 0.003$ and 0.244 \pm 0.007 derived respectively from the Keck II and MMT observations of the brightest $\mathrm{H}$ II region in SBS $0940+544$ are in good agreement with previous determinations of the helium abundance in this galaxy and are consistent with the value of the primordial ${ }^{4}$ He abundance $Y_{\mathrm{p}}=0.244-0.245$ derived by Izotov \& Thuan (1998b) and Izotov et al. (1999);

4. The hydrogen $\mathrm{H} \alpha$ and $\mathrm{H} \beta$ lines in the main body are seen in emission, while higher-order Balmer lines are seen in absorption. Three methods are used to constrain the age of the stellar populations in the main body of SBS $0940+544$. The first one is based on the age dependence of the $\mathrm{H} \alpha$ and $\mathrm{H} \beta$ emission line equivalent widths, the second one on the age dependence of the hydrogen $\mathrm{H} \gamma$ and $\mathrm{H} \delta$ absorption line equivalent widths, and the third one on the age dependence of the spectral energy distribution. Several star formation histories have been considered. The single instantaneous burst models do not reproduce the observed SEDs, suggesting that star formation in the main body was continuous. We find that models of continuous star formation with a constant star formation rate starting 10 Gyr ago are also excluded. However, models with continuous star formation during the period 0-10 Gyr, and with a varying star formation rate, are able to explain the observed properties. In particular, models in which the star formation rate during the last $100 \mathrm{Myr}$ has increased several times can reproduce both observed equivalent widths of the emission and absorption hydrogen lines and SEDs. However, the observed spectroscopic and photometric characteristics are reproduced equally well by models in which stars were continuously formed during the last $100 \mathrm{Myr}$ only, if 
an extinction correction as derived from the $\mathrm{H} \alpha / \mathrm{H} \beta$ flux ratio is applied to the SEDs;

5. The age of the reddest very low-intensity southern region in the SBS 0940+544 main body gives an upper limit to the galaxy's age. Because of the region's faintness, we were not able to detect hydrogen emission and/or absorption features to derive a reliable age. Assuming no extinction, we find that the SED can be fitted by a stellar population continuously formed with a constant star formation rate for a whole Hubble time. However, some extinction is likely to exist. In particular, if an extinction coefficient $C(\mathrm{H} \beta)=0.15$ is assumed, the extinction-corrected spectral energy distribution of the southern region can be well fitted by a model with a stellar population continuously formed with a constant star formation rate between $100 \mathrm{Myr}$ and 1 Gyr ago.

In summary, we find no compelling evidence which favours either a young or an old age for SBS 0940+544.

Acknowledgements. We thank the referee Göran Östlin for valuable comments and criticism on the manuscript. N. G. G. has been supported by DFG grant 436 UKR 17/1/00 and Y. I. I. acknowledges the Göttingen Academy of Sciences for a Gauss professorship. N. G. G. and Y. I. I. have been partially supported by INTAS 97-0033 and Swiss SCOPE 7UKPJ62178 grants. They are grateful for the hospitality of the Göttingen Observatory. Those authors, P. P. and K. J. F. acknowledge support by the Volkswagen Foundation under grant No. I/72919. Y. I. I. and T. X. T. have been partially supported by NSF grant AST-9616863. Research by P. P. and K. J. F. has been supported by the Deutsches Zentrum für Luft- und Raumfahrt e. V. (DLR) under grant 50 OR 9907 7. C. B. F. acknowledges the support of the NSF under grant AST-9803072, and K. G. N. of the Deutsche Forschungsgemeinschaft (DFG) grant FR325/50-1.

\section{References}

Aller, L. H. 1984, Physics of Thermal Gaseous Nebulae (Dordrecht: Reidel)

Anders, E., \& Grevesse, N. 1989, Geochim. Cosmochim. Acta, 53, 197

Augarde, R., Chalabaev, A., Comte, G., Kunth, D., \& Maehara, H. 1994, A\&AS, 104, 259

Bergvall, N., Rönnback, J., Masegosa, J., \& Östlin, G. 1999, A\&A, 341, 697

Bertelli, G., Bressan, A., Chiosi, C., Fagotto, F., \& Nasi, E. 1994, A\&AS, 106, 275

Bessell, M. S. 1990, PASP, 102, 1181

Bessell, M. S., Castelli, F., \& Plez, B. 1998, A\&A, 333, 231

Bica, E., \& Alloin, D. 1986, A\&A, 162, 21

Binggeli, B., \& Cameron, L. M. 1991, A\&A, 252, 27

Bohlin, R. C. 1996, AJ, 111, 1743

Brocklehurst, M. 1971, MNRAS, 153, 471

Cerviño, M., Luridiana, V., \& Castander, F. J. 2000, A\&A, 360, L5
Comte, G., Augarde, R., Chalabaev, A., Kunth, D., \& Maehara, H. 1994, A\&A, 285, 1

Crone, M. M., Schulte-Ladbeck, R. E., Hopp, U., \& Greggio, L. 2000, ApJ, 545, 31

Doublier, V., Caulet, A., \& Comte, G. 1999, A\&AS, 138, 213

Doublier, V., Comte, G., Petrosian, A., Surace, C., \& Turatto, M. 1997, A\&AS, 124, 405

Ferland, G. J. 1980, PASP, 92, 596

Filippenko, A. V. 1982, PASP, 94, 715

Fioc, M., \& Rocca-Volmerange, B. 1997, A\&A, 326, 950

Fricke, K. J., Izotov, Y. I., Papaderos, P., Guseva, N. G., \& Thuan, T. X. 2001, AJ, 121, 169

Garnett, D. R. 1992, AJ, 103, 1330

Gonzalez Delgádo, R. M., \& Leitherer, C. 1999, ApJS, 125, 479

Gonzalez Delgádo, R. M., Leitherer, C., \& Heckman T. M. 1999, ApJS, 125, 489

Henry, R. B. C., Edmunds, M. G., \& Köppen, J. 2000, ApJ, 541,660

Hunter, D. A., \& Thronson, H. A., Jr. 1995, ApJ, 452, 238

Izotov, Y. I., \& Thuan, T. X. 1998a, ApJ, 497, 227

Izotov, Y. I., \& Thuan, T. X. 1998b, ApJ, 500, 188

Izotov, Y. I., \& Thuan, T. X. 1999, ApJ, 511, 639

Izotov, Y. I., Chaffee, F. H., Foltz, C. B., et al. 1999, ApJ, 527, 757

Izotov, Y. I., Foltz, C. B., Green, R. F., Guseva, N. G., \& Thuan, T. X. 1997a, ApJ, 487, 37L

Izotov, Y. I., Lipovetsky, V. A., Chaffee, F. H., et al. 1997b, ApJ, 476, 698

Izotov, Y. I., Thuan, T. X., \& Lipovetsky, V. A. 1997c, ApJS, 108,1

Izotov, Y. I., Lipovetsky, V. A., Guseva, N. G., Kniazev, A. Y., \& Stepanian, J. A. 1991, A\&A, 247, 303

Izotov, Y. I., Thuan, T. X., \& Lipovetsky, V. A. 1994, ApJ, 435,647

Kunth, D., \& Östlin, G. 2000, A\&ARe, 10, 1

Kunth, D., \& Sargent, W. L. W. 1983, ApJ, 273, 81

Landolt, A. U. 1992, AJ, 104, 340

Lequeux, J., Rayo, J. F., Serrano, A., Peimbert, M., \& TorresPeimbert, S. 1979, A\&A, 80, 155

Lejeune, T., Cuisinier, F., \& Buser, R. 1998, A\&AS, 130, 65

Loose, H.-H., \& Thuan, T. X. 1986, in Star Forming Dwarf Galaxies and Related Objects, ed. D. Kunth, T. X. Thuan \& J. T. T. Van (Éditions Frontières), 73

Makarova, L. 1999, A\&A, 139, 491

Markarian, B. E., Lipovetsky, V. A., \& Stepanian, J. A. 1983, Afz, 19, 29

Mendoza, C. 1983, in IAU Symp. 103, Planetary Nebulae, ed. D. R. Flower (Dordrecht: Reidel), 143

Noeske, K. G., Guseva, N. G., Fricke, K. J., Izotov, Y. I., Papaderos, P., \& Thuan, T. X. 2000, A\&A, 361, 33

Oke, J. B. 1990, AJ, 99, 1621

Oke, J. B., Cohen, J. G., Carr, M., et al. 1995, PASP, 107, 375

Papaderos, P., Fricke, K. J., Thuan, T. X., Izotov, Y. I., \& Nicklas, H. 1999, A\&A, 352, L57

Papaderos, P., Izotov, Y. I., Fricke, K. J., Guseva, N. G., \& Thuan, T. X. 1998, A\&A, 338, 43

Papaderos, P., Loose, H.-H., Fricke, K. J., \& Thuan, T. X. 1996a, A\&A, 314, 59

Papaderos, P., Loose, H.-H., Thuan, T. X., \& Fricke, K. J. 1996b, A\&AS, 120, 207

Patterson, R. J., \& Thuan, T. X. 1996, ApJS, 107, 103

Pilyugin L. S. 1999, A\&A, 346, 428

Rönnback, J., \& Bergvall, N. 1994, A\&AS, 108, 193 
Sargent, W. L. W., \& Searle, L. 1970, ApJ, 162, L155

Schulte-Ladbeck, R. E., Crone, M. M., \& Hopp, U. 1998, ApJ, 493, L23

Sérsic, J. L. 1968, Atlas de Galaxias Australes (Córdoba: Obs. Astron.)

Stasińska G. 1990, A\&AS, 83, 501

Telles, E., Melnick, J., \& Terlevich, R. 1997, MNRAS, 288, 78

Telles, E., \& Terlevich, R. 1997, MNRAS, 286, 183

Thuan, T. X. 1983, ApJ, 268, 667

Thuan, T. X., \& Izotov, Y. I. 1997, ApJ, 489, 623

Thuan, T. X., \& Martin, G. E. 1981, ApJ, 247, 823

Thuan, T. X., Izotov, Y. I., \& Lipovetsky, V. A. 1995, ApJ, 445,108

Thuan, T. X., Izotov, Y. I., \& Lipovetsky, V. A. 1997, ApJ, 477,661
Thuan, T. X., Izotov, Y. I., \& Foltz, C. B. 1999a, ApJ, 525, 105

Thuan, T. X., Lipovetsky, V. A., Martin, J.-M., \& Pustilnik, S. A. 1999b, A\&AS, 139, 1

Thuan, T. X., Sauvage, M., \& Madden, S. 1999c, ApJ, 516, 783

Vacca, W. D., Garmany, C. D., \& Shull, J. M. 1996, ApJ, 460, 914

van Zee, L. 2000, AJ, 119, 2757

van Zee, L., Westphahl, D., Haynes, M., \& Salzer, J. J. 1998, AJ, 115, 1000

Vennik, J., Hopp, U., Kovachev, B., et al. 1996, A\&AS, 117, 216 